\title{
IllinoisGRMHD: an open-source, user- friendly GRMHD code for dynamical spacetimes
}

\author{
Zachariah B Etienne ${ }^{1,7}$, Vasileios Paschalidis ${ }^{2}$, \\ Roland Haas ${ }^{3}$, Philipp Mösta ${ }^{4}$ and Stuart L Shapiro ${ }^{5,6}$ \\ ${ }^{1}$ Department of Mathematics, West Virginia University, Morgantown, WV \\ 26506, USA \\ ${ }^{2}$ Department of Physics, Princeton University, Princeton, NJ 08544, USA \\ ${ }^{3}$ Max-Planck-Institut für Gravitationsphysik, Albert-Einstein-Institut, D-14476 Golm, \\ Germany \\ ${ }^{4}$ TAPIR, Mailcode 350-17, California Institute of Technology, Pasadena, CA \\ 91125, USA \\ ${ }^{5}$ Department of Physics, University of Illinois at Urbana-Champaign, Urbana, IL \\ 61801, USA \\ ${ }^{6}$ Department of Astronomy and NCSA, University of Illinois at Urbana-Champaign, \\ Urbana, IL 61801, USA \\ E-mail: zbetienne@mail.wvu.edu,vp16@princeton.edu,rhaas@aei.mpg.de, \\ pmoesta@tapir.caltech.edu and slshapir@illinois.edu
}

Received 28 January 2015, revised 26 May 2015

Accepted for publication 23 June 2015

Published 10 August 2015

\begin{abstract}
In the extreme violence of merger and mass accretion, compact objects like black holes and neutron stars are thought to launch some of the most luminous outbursts of electromagnetic and gravitational wave energy in the Universe. Modeling these systems realistically is a central problem in theoretical astrophysics, but has proven extremely challenging, requiring the development of numerical relativity codes that solve Einstein's equations for the spacetime, coupled to the equations of general relativistic (ideal) magnetohydrodynamics (GRMHD) for the magnetized fluids. Over the past decade, the Illinois numerical relativity (ILNR) group's dynamical spacetime GRMHD code has proven itself as a robust and reliable tool for theoretical modeling of such GRMHD phenomena. However, the code was written 'by experts and for experts' of the code, with a steep learning curve that would severely hinder community adoption if it were open-sourced. Here we present IllinoisGRMHD, which is an open-source, highly extensible rewrite of the original closed-source
\end{abstract}

7 Author to whom any correspondence should be addressed. 
GRMHD code of the ILNR group. Reducing the learning curve was the primary focus of this rewrite, with the goal of facilitating community involvement in the code's use and development, as well as the minimization of human effort in generating new science. IllinoisGRMHD also saves computer time, generating roundoff-precision identical output to the original code on adaptive-mesh grids, but nearly twice as fast at scales of hundreds to thousands of cores.

Keywords: GRMHD, magnetohydrodynamics, general relativity, black holes, neutron stars, gamma-ray bursts, relativistic astrophysics

(Some figures may appear in colour only in the online journal)

\section{Introduction and motivation}

Incident gravitational wave $(\mathrm{GW})$ observations have the potential to address some of the most important unsolved problems in astronomy and theoretical astrophysics. These include testing GR in the strong field regime, determining the engine behind short-hard gamma-ray bursts, constraining the equation of state above nuclear densities, revealing how compact binaries form and evolve, as well as uncovering the distributions of black hole (BH) spins and masses, just to name a few. However, it is well known that unless some coincident electromagnetic (EM) counterpart from the GW source is observed, GW interferometers alone may be unable to pinpoint the source position on the sky, hindering parameter estimation [35, 45, 51, 64]. Moreover, EM signals carry additional and complementary information about the source, lending potentially critical insights about the GW source and its environment.

Thus detections of EM counterparts to GWs could be critically important in this age of 'multimessenger' astronomy, and not solely when GWs are detected first. For example, it may be possible that an EM signal itself would imply a GW source, leading to targeted searches across the GW spectrum, from the $\mathrm{nHz}$ band in the case of dual AGNs, to the $\mathrm{kHz}$ band in the case of stellar-mass binaries and supernovae. Beyond coincident GW detections, EM transients linked to strong-field, dynamical spacetime phenomena may themselves greatly advance our understanding of $\mathrm{BH}$ accretion phenomena and matter at extreme densities.

However, without detailed theoretical models of EM counterparts to GW observations, our interpretation of observed EM counterparts may be severely limited. Constructing such models remains a central problem in theoretical astrophysics, for two key reasons. First, observable signals are often sensitive to fluid flows and gravitational fields spanning many orders of magnitude in lengthscale and timescale. Second, the equations governing the dynamics are highly complex and nonlinear, requiring the evolution of the full set of Einstein's equations of general relativity, coupled to the equations of general relativistic magnetohydrodynamics (GRMHD).

Thus numerical relativity codes capable of modeling multi-scale GRMHD flows promise to not only provide key insights into these important phenomena, but represent the starting point for more sophisticated modeling that includes advanced EM and neutrino radiation transport. More than a decade ago, the Illinois numerical relativity (ILNR) group led by Shapiro was among the first to develop a dynamical spacetime GRMHD code [25] for uniform-resolution grids. Since then, this GRMHD code (henceforth, OrigGRMHD) has been significantly extended and improved. Its current version models multi-scale GRMHD flows via an adaptive-mesh-refinement (AMR) vector-potential formulation. By evolving the vector potential forward in time instead of the magnetic fields directly, this formulation guarantees 
the no-monopole constraint $\nabla \cdot \boldsymbol{B}=0$ is satisfied over the entire numerical grid, even when multi-scale magnetized fluid flows cross AMR grid boundaries. Notably, this formulation reduces to the standard, staggered flux-constrained-transport [11] scheme on uniform-resolution numerical grids [30, 32, 36].

OrigGRMHD's reputation for generating models that address key unsolved problems in theoretical astrophysics has been built upon years of hard-fought development, as there exists no standard, proven algorithms for dynamical spacetime, multi-scale GRMHD modeling. Over the past decade, the code has been used to model a number of astrophysical scenarios, gleaning key new insights into these systems. For example, OrigGRMHD has produced stateof-the-art magnetized binary neutron stars (NSs) [46, 56] and binary BH-NS $[27,28,31,33,59]$ models. It was also used to simulate magnetized disk accretion onto binary BHs [36, 37, 40, 41], binary white dwarf-NS mergers [55, 58], magnetized, rotating NSs [29], magnetized Bondi accretion [30], and magnetized hypermassive NSs $[22,23,47,65,66,68]$, just to name a few. The code was also recently extended, as a separate module, to solve the equations of GR force-free electrodynamics [60] and applied to model both binary BH-NS [57] and pulsar magnetospheres in GR [61]. At each stage of its development, OrigGRMHD was subjected to a large battery of stringent test-bed problems [30], which it had to pass before being used for applications.

The field has matured considerably in the years since the first dynamical spacetime GRMHD codes were announced, and multiple groups now possess their own independent codes $[2,16,21,25,30,32,39,44,50,54,63]$, most of which solve these equations on AMR grids. Given the time and effort required to extend such codes to model more physics, while still maintaining and improving the GRMHD modules, it seems clear that the community might benefit if more of us consolidated our efforts and adopted the same dynamic-spacetime GRMHD code.

With its proven robustness and reliability in modeling some of the most extreme phenomena in the Universe, OrigGRMHD appears to be a good candidate for such community adoption if it were open-sourced. But despite its strong track record, OrigGRMHD was not written with community adoption in mind, instead being a code written by experts and for experts' of the code. As such, the code lacked a number of features common to top-notch, widely adopted open-source projects in computational astrophysics, including sufficient documentation and code comments, fine-grained modularity, a consistent coding style, and regular, enforced code maintenance (e.g., removal of unused and unmaintained features).

Thus the OrigGRMHD core development team came to the understanding that unless these idiosyncrasies were fixed, open-sourcing the code would be unlikely to engender widespread community adoption. Thus in early 2013, it was decided to rewrite OrigGRMHD from the ground up, with a focus on the four core design principles of user-friendliness, robustness, modularity/extensibility, and performance/scalability. Slightly more than a year later, all of OrigGRMHD's core algorithms had been rewritten and the new code, IllinoisGRMHD, was released.

Just after the decision was made to rewrite OrigGRMHD in 2013, the first open-source, dynamical spacetime GRMHD code, called GRHydro, was released [50]. Originally forked from the dynamical spacetime, general relativistic hydrodynamics Whisky code [8] (not to be confused with its closed-source successor, WhiskyMHD [39]), GRHydro shares many of the same features of OrigGRMHD, including a number of reconstruction techniques.

However, unlike OrigGRMHD/IllinoisGRMHD, GRHydro's GRMHD scheme has not been developed to forbid the generation of monopoles (i.e., violations of the $\nabla \cdot \boldsymbol{B}=0$ constraint) when magnetized fluids flow across AMR grid boundaries. As accurate modeling of such multi-scale fluid flows is critically important in many astrophysical scenarios of 
interest to the community, GRHydro's adoption by members of the community has been limited, primarily to those who simulate core collapse.

Further, one of OrigGRMHD/IllinoisGRMHD's key advantages is that these codes are capable of stably modeling GRMHD flows into BH horizons over very long timescales, without the need for special algorithms that excise GRMHD data within the BH. By contrast, it seems that $\mathrm{BH}$ excision is an essential ingredient for stable GRMHD evolutions with GRHydro in the presence of BHs. To date GRHydro has been mostly used for core collapse (to a NS) simulations, in which no BH is present.

OrigGRMHD/IllinoisGRMHD have been demonstrated robust across a much wider range of long-term $\mathrm{BH}$ simulations, and manage to do so without excision. We conclude that making GRHydro's GRMHD schemes as robust may require careful specification of boundary conditions on the excision surface coupled to an interpolation scheme across AMR level boundaries that respects the no-monopoles constraint, e.g. [9, 10].

IllinoisGRMHD was originally designed in a standalone sandbox to maximize portability to other parallel infrastructures, but currently adopts the latest Einstein Toolkit (ET) [48]/ Carpet [62] AMR infrastructure. IllinoisGRMHD has been proposed for inclusion within the next ET release, and code review is underway. In the meantime, the ET community have graciously agreed to host the IllinoisGRMHD code, in anticipation of official incorporation upon completion of the code review process ${ }^{8}$.

In this paper, we present results from a number of code validation tests demonstrating that IllinoisGRMHD (1) produces results identical to OrigGRMHD, (2) possesses identical or significantly better scalability and performance than OrigGRMHD and GRHydro, (3) generates results in quantitative agreement with those of the GRHydro code, in the context of dynamical spacetime evolutions of Tolman-Oppenheimer-Volkoff (TOV) stars.

The remainder of the paper is organized as follows. Section 2 outlines the formulation of the GRMHD equations solved by IllinoisGRMHD, section 3 presents a basic overview of algorithms used within IllinoisGRMHD, section 4 shows results from code validation tests, section 5 demonstrates the outstanding performance and scalability of IllinoisGRMHD via benchmarks, and section 6 summarizes results and describes future work.

\section{Basic equations}

All equations presented below are in geometrized units where $G=c=1$. In these units, Einstein's equations become

$$
G^{\mu \nu}=8 \pi T^{\mu \nu},
$$

where $G^{\mu \nu}$ is the Einstein tensor and $T^{\mu \nu}$ the total stress-energy tensor. IllinoisGRMHD solves the coupled Einstein-Maxwell equations assuming a perfect fluid stress-energy tensor for the matter and infinite conductivity (ideal MHD), by evolving via high-resolution-schockcapturing techniques the GRMHD quantities that comprise the stress-energy tensor $T^{\mu \nu}$, acting as the source for Einstein's equations. With these assumptions, the GRMHD evolution and constraint equations are derived from the following basic equations:

(i) Conservation of baryon number

$$
\nabla_{\mu}\left(\rho_{0} u^{\mu}\right)=0
$$

\footnotetext{
${ }^{8}$ Instructions for downloading, compiling, and using IllinoisGRMHD may be found here: http://math.wvu.edu/ $\sim$ zetienne/ILGRMHD/.
} 
where $\nabla_{\mu}$ is the covariant derivative associated with the spacetime metric tensor $g_{\mu \nu}, \rho_{0}$ is the fluid rest-mass density and $u^{\mu}$ is the fluid four-velocity.

(ii) Conservation of energy-momentum

$$
\nabla_{\mu} T^{\mu \nu}=0, T_{\mu \nu}=T_{\mu \nu}^{\mathrm{matter}}+T_{\mu \nu}^{\mathrm{EM}}
$$

where $T_{\mu \nu}$ is the sum of the perfect fluid $T_{\mu \nu}^{\text {matter }}$ and EM stress-energy tensors $T_{\mu \nu}^{\mathrm{EM}}$ in the ideal MHD limit $\left(u_{\mu} F^{\mu \nu}=0\right)$.

(iii) Homogeneous Maxwell's equations

$$
\nabla_{\nu} F^{* \mu \nu}=\frac{1}{\sqrt{-g}} \partial_{\nu}\left(\sqrt{-g} F^{* \mu \nu}\right)=0,
$$

where $F^{\mu \nu}$ the Faraday tensor, $F^{* \mu \nu}=(1 / 2) \epsilon^{\mu \nu \rho \sigma} F_{\rho \sigma}$ its dual $\left(\epsilon^{\mu \nu \rho \sigma}\right.$ is the Levi-Civita tensor), and $g$ the determinant of $g_{\mu \nu}$.

As written, equations (2)-(4) for the plasma, as well as equation (1) for the spacetime metric, are not particularly well-suited for numerical evolutions, so we choose special formulations of them. For the spacetime metric evolution, we choose an initial value formulation built upon first splitting the four-metric $g_{\mu \nu}$ into the standard 3+1 Arnowitt-Deser-Misner (ADM) form [5]:

$$
\mathrm{d} s^{2}=g_{\mu \nu} \mathrm{d} x^{\mu} \mathrm{d} x^{\nu}=-\alpha^{2} \mathrm{~d} t^{2}+\gamma_{i j}\left(\mathrm{~d} x^{i}+\beta^{i} \mathrm{~d} t\right)\left(\mathrm{d} x^{j}+\beta^{j} \mathrm{~d} t\right)
$$

Here, $\alpha$ is the lapse function, $\beta^{i}$ the shift vector, and $\gamma_{i j}$ the three-metric on spacelike hypersurfaces of constant time $t$. This basic decomposition of the four-metric can be used to split the Einstein equations (1) into a set of evolution equations and a set of constraint equations that the dynamical variables must satisfy for all times-similar to Maxwell's equations-with projections of $T^{\mu \nu}$ along and normal to the 3D spatial hypersurface existing as source terms (see, e.g., [14] for a detailed discussion and references). This original formulation of the Einstein equations is known as the ADM 3+1 formulation of GR. A number of $3+1$ formulations can be derived from the ADM formulation and are useful for solving the Cauchy problem for the Einstein equations. For the purposes of this paper, we choose the Baumgarte-Shapio-Shibata-Nakamura (BSSN) formulation [13, 67], which introduces an auxiliary dynamical variable and conformal scalings for the dynamical variables, casting the evolution equations in a form that allows for stable, long-term and accurate numerical integration.

To update $T^{\mu \nu}$ from one time slice to the next in a simulation, IllinoisGRMHD solves equations (2), (3), and the spatial component of equation (4) in the ideal MHD limit $\left(u_{\mu} F^{\mu \nu}=0\right)$, as written in conservative form (see e.g. [25]):

$$
\partial_{t} \boldsymbol{C}+\nabla \cdot \boldsymbol{F}=\boldsymbol{S}
$$

where $\boldsymbol{F}$ is the flux vector, $\boldsymbol{C}=\left\{\rho_{\star}, \tilde{\tau}, \tilde{S}_{i}, \tilde{B}^{i}\right\}$ the vector of conservative variables, and $\boldsymbol{S}$ the vector of source terms These vectors depend directly on the 'primitive' variables $\boldsymbol{P}=\left\{\rho_{0}, P, v^{i}, B^{i}\right\}$, where $P$ is the pressure, $v^{i}=u^{i} / u^{0}$ the fluid three-velocity, and $B^{i}$ are the spatial components of the magnetic field $\left(B^{\mu}\right)$ measured by normal (or Eulerian) observers with four velocity $n^{\mu}=\left(1,-\beta^{i}\right) / \alpha$ (and is normal to the spatial hypersurface, $B^{\mu} n_{\mu}=0$, as well as the metric and its derivatives. In particular, $\boldsymbol{C}$ may be written in terms of $\boldsymbol{P}, \alpha$ and the metric as follows: 


$$
\boldsymbol{C}=\left[\begin{array}{c}
\rho_{\star} \\
\tilde{\tau} \\
\tilde{S}_{i} \\
\tilde{B}^{i}
\end{array}\right]=\left[\begin{array}{c}
\alpha \sqrt{\gamma} \rho_{0} u^{0} \\
\alpha^{2} \sqrt{\gamma} T^{00}-\rho_{\star} \\
\left(\rho_{\star} h+\alpha u^{0} \sqrt{\gamma} b^{2}\right) u_{i}-\alpha \sqrt{\gamma} b^{0} b_{i} \\
\sqrt{\gamma} B^{i},
\end{array}\right],
$$

where $\gamma$ is the determinant of the three-metric $\gamma_{i j}, h=1+\epsilon+P / \rho_{0}$ is the specific enthalpy, with $\epsilon$ the specific internal energy, and $b^{\mu}=B_{(u)}^{\mu} / \sqrt{4 \pi}$ with $B_{(u)}^{\mu}$ the magnetic field field measured by an observer comoving with the fluid. Here the total stress-energy tensor $T^{\mu \nu}$ can be written as follows (see [14, 24, 25] for further details and derivations)

$$
T^{\mu \nu}=\left(\rho_{0} h+b^{2}\right) u^{\mu} u^{\nu}+\left(P+\frac{b^{2}}{2}\right) g^{\mu \nu}-b^{\mu} b^{\nu} .
$$

Our choice of fluid three-velocity $v^{i}=u^{i} / u^{0}$ as a primitive variable is consistent with a number of GRMHD codes, such as [26, 44, 53]. However, our $v^{i}$ differs from that of other GRMHD codes (e.g., $[6,7,39,50]$, just to name a few) that have adopted the Valencia formalism [4, 12], which adopts the fluid three-velocity $v_{(n)}^{i}$ as measured by normal observers (also referred to as the Eulerian three-velocity), defined as:

$$
\begin{aligned}
& u^{a}=\alpha u^{0}\left(n^{a}+v_{(n)}^{a}\right) \Rightarrow \\
& v_{(n)}^{i}=\frac{u^{i}}{\alpha u^{0}}+\frac{\beta^{i}}{\alpha} .
\end{aligned}
$$

Note that $v_{(n)}^{\mu}$ is orthogonal to the normal vector to the 3D spatial hypersurface $v_{(n)}^{\mu} n_{\mu}=0$. In terms of the fluid three-velocity used by IllinoisGRMHD $v^{i}, v_{(n)}^{i}$ can be written as

$$
v_{(n)}^{i}=\frac{1}{\alpha}\left(v^{i}+\beta^{i}\right) .
$$

Note that this difference in the three-velocity variable may account for some of the differences in numerical results observed between IllinoisGRMHD and (the Valencia-based) GRHydro in section 4, as Valencia-based codes reconstruct $v_{(n)}^{i}$ instead of $v^{i}$.

Writing the GRMHD evolution equations in conservative form offers a number of numerical advantages. First, when the source terms $\mathbf{S}$ vanish (e.g. in Minkowski spacetime), it guarantees conservation of total rest mass $\left(\int_{V} \rho_{*} \mathrm{~d}^{3} x\right)$, energy $\left(\int_{V} \tilde{\tau} \mathrm{d}^{3} x\right)$, and momentum $\left(\int_{V} \tilde{S}_{i} \mathrm{~d}^{3} x\right)$ to roundoff error. When the source terms are accounted for, total ADM mass and momentum are conserved to within truncation error. Second, it enables us to attach easily an approximate Riemann solver, yielding a state-of-the-art high-resolution shock-capturing (HRSC) numerical scheme, designed in part to minimize oscillations near shocks. Such oscillations are generated by approximating the flux derivative across shocks by smooth functions. Third, the conservative form, coupled to the HRSC scheme guarantees the shock jump conditions (Rankine-Hugoniot) are satisfied. From an empirical perspective, finitevolume conservative formulations, as implemented in IllinoisGRMHD, have been shown superior at handling ultrarelativistic flows when compared to advanced artificial viscosity (AV) schemes [3, 49], despite the fact that AV schemes are typically superior in terms of ease of implementation and computational efficiency.

A key ingredient in a robust GRMHD code is the proper treatment of the magnetic induction equation, which is derived from the spatial components of equation (4). In 
conservative form, these may be written:

$$
\partial_{t} \tilde{B}^{i}+\partial_{j}\left(v^{j} \tilde{B}^{i}-v^{i} \tilde{B}^{j}\right)=0
$$

If the induction equation is directly evaluated in a numerical code without special techniques, numerical truncation errors that violate the divergence-free or 'no-monopoles' constraint

$$
\partial_{i} \tilde{B}^{i}=0
$$

will be generated. Note that this equation is simply the time component of equation (4). Maintaining satisfaction of this constraint as the magnetic fields are evolved forward in time (through direct evaluation of equation (12)) happens to be a nontrivial endeavor, particularly on AMR grids. Our solution [30,32] is to evolve the magnetic four-vector potential $\mathcal{A}_{\mu}$ instead of the magnetic fields directly, so that

$$
\begin{aligned}
& \mathcal{A}_{\mu}=\Phi n_{\mu}+A_{\mu}, \text { and } \\
& \tilde{B}^{i}=\tilde{\epsilon}^{i j k} \partial_{j} A_{k},
\end{aligned}
$$

where $A_{\mu}$ is purely spatial $\left(A_{\mu} n^{\mu}=0\right)$ and $\Phi$ is the EM scalar potential. Here, $\tilde{\epsilon}^{i j k}$ is the standard permutation symbol, equal to $1(-1)$ if $i j k$ are an even (odd) permutation of 123 , and 0 if one or more indices are identical. Special finite difference operators for the vector potential are defined in IllinoisGRMHD so that the divergence of a curl is zero to roundoff error, which implies that the divergence of equation (15) is zero and equation (13) is satisfied automatically, even on AMR grids.

In terms of $A_{i}$, the induction equation (12) becomes

$$
\partial_{t} A_{i}=\tilde{\epsilon}_{i j k} v^{j} \tilde{B}^{k}-\partial_{i}\left(\alpha \Phi-\beta^{j} A_{j}\right) .
$$

What remains is to choose an EM gauge, and IllinoisGRMHD chooses the 'generalized Lorenz gauge condition' by default that was introduced by the Illinois Relativity group in [36]. The covariant version of the condition is $\nabla_{\mu} \mathcal{A}^{\mu}=\xi n_{\mu} \mathcal{A}^{\mu}$, where $\xi$ is a parameter with dimensions $1 /$ Length, chosen carefully so that the Courant-Friedrichs-Lewy factor remains satisfied. Typically $\xi$ is set to $1.5 / \Delta t_{\max }$, where $\Delta t_{\max }$ is the timestep of the coarsest refinement level. This gauge choice therefore yields the additional evolution equation

$$
\partial_{t}[\sqrt{\gamma} \Phi]+\partial_{j}\left(\alpha \sqrt{\gamma} A^{j}-\beta^{j}[\sqrt{\gamma} \Phi]\right)=-\xi \alpha \sqrt{\gamma} \Phi .
$$

Note that IllinoisGRMHD evolves not $\Phi$ but $\sqrt{\gamma} \Phi$ as the EM gauge variable.

With the exception of this purely gauge evolution equation and the vector-potential induction equation, all other GRMHD evolution equations are written in conservative form and are solved via a HRSC scheme, as described in section 3. For completeness, the remaining set of evolution equations evolved by IllinoisGRMHD are written in conservative form (equation (6)) as follows

$$
\partial_{t}\left[\begin{array}{c}
\rho_{\star} \\
\tilde{\tau} \\
\tilde{S}_{i}
\end{array}\right]+\partial_{j}\left[\begin{array}{c}
\rho_{\star} v^{j} \\
\alpha^{2} \sqrt{\gamma} T^{0 j}-\rho_{\star} v^{j} \\
\alpha \sqrt{\gamma} T^{j}{ }_{i}
\end{array}\right]=\left[\begin{array}{c}
0 \\
s \\
\frac{1}{2} \alpha \sqrt{\gamma} T^{\alpha \beta} g_{\alpha \beta, i}
\end{array}\right],
$$


where

$$
s=\alpha \sqrt{\gamma}\left[\left(T^{00} \beta^{i} \beta^{j}+2 T^{0 i} \beta^{j}+T^{i j}\right) K_{i j}-\left(T^{00} \beta^{i}+T^{0 i}\right) \partial_{i} \alpha\right],
$$

and $K_{i j}=-\mathfrak{£}_{n} \gamma_{i j} / 2$ is the extrinsic curvature, where $£_{n}$ designates the Lie derivative along the hypersurface normal vector $n$ (see e.g. [14] for more details).

Finally, to close the system of equations, the EOS of the matter must be specified. IllinoisGRMHD currently implements a hybrid EOS of the form [43]

$$
P\left(\rho_{0}, \epsilon\right)=P_{\text {cold }}\left(\rho_{0}\right)+\left(\Gamma_{\text {th }}-1\right) \rho_{0}\left[\epsilon-\epsilon_{\text {cold }}\left(\rho_{0}\right)\right],
$$

where $P_{\text {cold }}$ and $\epsilon_{\text {cold }}$ denote the cold component of $P$ and $\epsilon$ respectively, and $\Gamma_{\text {th }}$ is a constant parameter which determines the conversion efficiency of kinetic to thermal energy at shocks. The function $\epsilon_{\text {cold }}\left(\rho_{0}\right)$ is related to $P_{\text {cold }}\left(\rho_{0}\right)$ by the first law of thermodynamics

$$
\epsilon_{\text {cold }}\left(\rho_{0}\right)=\int \frac{P_{\text {cold }}\left(\rho_{0}\right)}{\rho_{0}^{2}} \mathrm{~d} \rho_{0} .
$$

All functions within IllinoisGRMHD support piecewise-defined $P_{\text {cold }}\left(\rho_{0}\right)$ (the so-called 'piecewise polytrope' EOS) with up to nine different polytropic indices, except for the conservatives-to-primitives solver, which currently supports only one.

In all code tests presented in this paper, the $\Gamma$-law EOS $P=(\Gamma-1) \rho_{0} \epsilon$ is adopted. This corresponds to setting $P_{\text {cold }}=(\Gamma-1) \rho_{0} \epsilon_{\text {cold }}$ in equation (20), which is equivalent to $P_{\text {cold }}=\kappa \rho_{0}^{\Gamma}$ (with constant $\kappa$ ), and $\Gamma_{\text {th }}=\Gamma$. In the absence of shocks and in the initial data used for our tests, $\epsilon=\epsilon_{\text {cold }}$ and $P=P_{\text {cold }}$.

\subsection{Outer boundary conditions}

We apply outer boundary conditions to primitive variables $\rho_{0}, P$, and $v^{i}$, which enforce a zero-derivative, 'copy' boundary condition of these quantities at the outer boundary, except when this results in a positive incoming velocity from the outer boundary. Hence we refer to these as 'outflow' boundary conditions. As our outer boundary exists as a rectangular box, if for example $v^{x}<0$ at a given point on the $x=x_{\max }$ boundary plane after applying the zeroderivative boundary condition, we set $v^{x}=0$ at that point. Similarly, at a given point on the $x=x_{\min }$ boundary plane, if $v^{x}>0$ after applying the flat outer boundary condition, $v^{x}$ is set to zero. The same strategy is applied to the velocities for all other outer boundary faces.

We also apply outer boundary conditions to $A_{\mu}$, linearly extrapolating values to the outer boundary. To avoid problems caused by reflections of EM gauge waves from these imperfect outer boundary conditions, the unit-bearing (i.e., not dimensionless) $\xi$ parameter in the $A_{i}$ evolution equation is set to some nonzero, positive value, typically $1.5 / \Delta t_{\max }$, where $\Delta t_{\max }$ is the timestep of the coarsest refinement level.

\section{Basic algorithms}

Since its initial stages, one of the primary objectives driving the development of IllinoisGRMHD has been to remove nonrobust algorithms and obsolete code from the original GRMHD code of the Illinois group, resulting in a reliable state-of-the-art piece of software that is more compact and easier for beginners to learn and extend. To this end, all of the obsolete code and functionality proven to be nonrobust in typical dynamical spacetime evolutions has been stripped from the code, keeping within IllinoisGRMHD only the set of algorithms used in all of the Illinois group's latest GRMHD publications. Further, the core 
algorithms themselves have been rewritten into a uniform coding standard, with large amounts of duplicated functionality replaced with a small, optimized library of functions. This section reviews the basic algorithms that comprise IllinoisGRMHD.

Here the algorithms that comprise the basic components of IllinoisGRMHD are introduced, in the order in which they are called. At the beginning of the first timestep, the variables $\left\{P, \rho_{0}, v^{i}, B^{i}, A_{\mu}, \Phi\right\}$ must be defined at every gridpoint. The following outlines the basic steps in which these variables are updated at all gridpoints, in preparation for the next timestep. All updates are performed by IllinoisGRMHD unless otherwise specified.

(i) First, the flux and right-hand side (rhs) terms in equations (17) and (18), for the set of evolution variables $\boldsymbol{E}=\left\{\rho_{*}, \tilde{S}_{i}, \tilde{\tau}, A_{i},[\sqrt{\gamma} \Phi]\right\}$, are evaluated. Three separate algorithms are employed in this step:

(a) A HRSC evolution scheme is used to compute the flux terms of the $\rho_{*}, \tilde{S}_{i}$, and $\tilde{\tau}$ evolution equations, as defined in equation (18). This scheme, as well as the technique used to compute the source terms related to spacetime curvature in these equations, is described in section 3.1.

(b) Unlike the other primitive variables, $A_{i}$ and $B^{i}$ are defined on staggered gridpoints. Further, our $A_{i}$ evolution scheme is constructed to produce identical output to the standard, staggered constrained transport scheme of [34]. As detailed in section 3.2, this makes the HRSC scheme for updating $A_{i}$ a bit more involved than the HRSC scheme for evolving the unstaggered densitized density, momentum, and energy variables.

(c) The evolution of the (staggered) EM gauge quantity $[\sqrt{\gamma} \Phi]$ is not based on a HRSC scheme, as this quantity generally does not exhibit sharp features in our simulations. Its evolution algorithm is summarized in section 3.3.

(ii) Time derivative data from all evolved GRMHD variables are then passed to the method of lines (MoL) thorn, which iteratively integrates the evolved variables forward in time. MoL is capable of managing a number of iterative, explicit time integration techniques, of which we typically choose the four-iteration Runge-Kutta fourth-order (RK4) scheme, both in IllinoisGRMHD and the chosen spacetime evolution thorn.

(iii) Evaluating the time derivatives of all evolved GRMHD variables requires three ghostzones at the outer boundary of each AMR grid. The ghostzones at the outermost boundary are filled at each RK4 iteration, using the outer boundary update procedure outlined in the next steps. However, ghostzones at each internal AMR grid boundary are allowed to accumulate until the end of the fourth RK4 iteration. Since RK4 consists of four iterations, this yields a total of $3 \times 4=12$ AMR grid boundary ghostzones that must be filled at the end of each full RK4 timestep. To fill these 12 ghostzones for all evolved variables at the end of the fourth RK4 iteration, prolongation and restriction operators are applied, which interpolate between different levels of refinement in both space and time. Third-order, line-averaged Lagrange prolongation/restriction is performed on $A_{i}$ and $\sqrt{\gamma} \Phi$, and fifth-order Lagrange prolongation/restriction is performed on all other GRMHD evolved variables.

(iv) Next, linear-extrapolation outer boundary conditions are applied to $A_{i}$ and $[\sqrt{\gamma} \Phi]$, as described in section 3.5. Then $B^{i}$ is computed from $A_{i}$ at all gridpoints, as described in section 3.2.

(v) The conservative variables $\rho_{*}, \tilde{S}_{i}, \tilde{\tau}$, and $\tilde{B}^{i}$ have at this point been updated at all needed gridpoints, except at the outer boundary. However, the primitive variables $\left\{P, \rho_{0}, v^{i}, B^{i}\right\}$ do not yet exist at any gridpoints. As these variables are required at the outset of the next iteration, a conservative-to-primitives solver is called next, which at its heart employs a 
Newton-Raphson-based root-finder to invert equation (7), computing primitive variables at each gridpoint based on the conservative variables at that point. Additionally, there are a number of consistency checks applied both before and after this solver is called. The procedure is outlined in section 3.4.

(vi) Next, zero-slope, outflow outer boundary conditions are applied to the set of primitive variables $\left\{P, \rho_{0}, v^{i}\right\}$, as described in section 3.5. After this step, all variables $\left\{P, \rho_{0}, v^{i}, B^{i}, A_{\mu}\right\}$ needed to repeat this process have been defined at all gridpoints, so to proceed to the next timestep or RK4 iteration, we simply loop to (i)(a). Values for the conservatives at the outer boundary are not strictly required for the evolution, but are set anyway, based on the primitive variables, in case a diagnostics utility might require that conservatives be set at the outer boundary.

To conclude this introduction to IllinoisGRMHD's basic algorithms, section 3.6 describes how IllinoisGRMHD connects to the rest of the ET and its spacetime metric evolution modules.

\subsection{Evolution of $\rho_{*}, \tilde{S}_{i}$, and $\tilde{\tau}$}

To evolve the GRMHD variables, IllinoisGRMHD first evaluates the source terms of the time evolution equations for $\left\{\rho_{*}, \tilde{S}_{i}, \tilde{\tau}\right\}$ in equation (18). Derivatives of the spacetime fields appear in the source terms, which are evaluated via standard, second-order (default) or fourth-order finite differences. Next, the fluxes are computed via a second-order finite-volume HRSC scheme. Since point values of gridfunctions and their volume averages are the same to second order, our finite-volume scheme will converge at the same order as a finite-difference scheme. We choose a finite-volume scheme, as it will enable us to more rapidly move to a higher-order method in future releases of IllinoisGRMHD, following the strategy outlined in [30].

Computation of the flux term $\nabla \cdot \boldsymbol{F}=\partial_{m} F^{m}$ in a given direction $i \in\{x, y, z\}$ is performed with our second-order finite-volume scheme in two steps, as detailed below. First, the Reconstruction step computes values for the primitive variables at cell interfaces (between gridpoints) along direction $i$. Then the Riemann solver solves the Riemann problem via an inexpensive, approximate algorithm, ensuring the conservative variable fluxes between gridpoints are appropriately constructed along direction $i$, even in the presence of discontinuities or shocks. Upon completing the Riemann solver step for a given flux direction $i$, the process is repeated in the other two directions until $\partial_{m} F^{m}$ has been evaluated and summed in all three spatial dimensions $\{x, y, z\}$.

The reconstruction step for $\rho_{*}, \tilde{S}_{i}$, and $\tilde{\tau}$ : IllinoisGRMHD employs the piecewise parabolic method (PPM) [17], incorporating the original flattening and steepening procedures to reconstruct $\boldsymbol{P}$ at the right $\left(\boldsymbol{P}_{\boldsymbol{R}}\right)$ and left $\left(\boldsymbol{P}_{\boldsymbol{L}}\right)$ sides of each grid zone interface, along direction $i \in\{x, y, z\}$. The version of PPM used within IllinoisGRMHD is designed to maintain thirdorder accuracy, except at discontinuities or shocks and at local minima and maxima. As in the GRHydro code [50] the flattening procedure within PPM was simplified to decrease the number of required ghostzones within PPM from four to three.

After PPM reconstruction evaluates $\boldsymbol{P}_{\boldsymbol{R}, \boldsymbol{L}}$ along a given direction $i$ and the metric values have been interpolated to each grid zone interface at fourth-order (default) or second-order accuracy, the fluxes $\boldsymbol{F}_{\boldsymbol{R}, \boldsymbol{L}}$ are then immediately evaluated via (7) and equation (18).

Next, for appropriate handling of fluxes across a given cell interface, the Riemann problem must be solved.

The Riemann solver for $\rho_{*}, \tilde{S}_{i}$, and $\tilde{\tau}$ : the first step in solving the Riemann problem along direction $i \in\{x, y, z\}$ is to compute the maximum (+) and minimum (-) characteristic speeds 
$c_{ \pm}^{\mathrm{R}, \mathrm{L}}$ at each cell interface, approximating the general GRMHD dispersion relation (equation (27) of [38]) with the following, simpler expression:

$$
\omega_{\mathrm{cm}}^{2}=\left[v_{\mathrm{A}}^{2}+c_{\mathrm{s}}^{2}\left(1-v_{\mathrm{A}}^{2}\right)\right] k_{\mathrm{cm}}^{2} .
$$

Here, $\omega_{\mathrm{cm}}=-k_{\mu} u^{\mu}$ is the frequency and $k_{\mathrm{cm}}^{2}=K_{\mu} K^{\mu}$ the wavenumber of an MHD wave mode in the frame comoving with the fluid, where $K_{\mu}$ is defined as the projection of the wave vector $k^{\nu}$ onto the direction normal to $u^{\nu}: K_{\mu}=\left(g_{\mu \nu}+u_{\mu} u_{\nu}\right) k^{\nu} . c_{\mathrm{S}}$ is the sound speed, and $v_{\mathrm{A}}$ is the Alfvén speed, given by

$$
v_{\mathrm{A}}=\sqrt{\frac{b^{2}}{\rho_{0} h+b^{2}}} .
$$

With these definitions, the approximate dispersion relation (equation (22)) may then be solved along direction $i$, noting that the wave vector along this direction in the comoving frame is given by $k_{\mu}=\left(-\omega, k_{j} \delta_{i}{ }^{j}\right)$ and the wave (phase) velocity by $c_{ \pm}=\omega /\left(k_{j} \delta_{i}{ }^{j}\right)$. The dispersion relation can then be written as a quadratic equation for $c_{ \pm}: a_{1} c_{ \pm}^{2}+a_{2} c_{ \pm}+a_{3}=0$, where

$$
\begin{aligned}
& a_{1}=\left(1-v_{0}^{2}\right)\left(u^{0}\right)^{2}-v_{0}^{2} g^{00}, \\
& a_{2}=2 v_{0}^{2} g^{i 0}-2 u^{i} u^{0}\left(1-v_{0}^{2}\right), \\
& a_{3}=\left(1-v_{0}^{2}\right)\left(u^{i}\right)^{2}-v_{0}^{2} g^{i i},
\end{aligned}
$$

and $v_{0}^{2}=v_{\mathrm{A}}^{2}+c_{\mathrm{s}}^{2}\left(1-v_{\mathrm{A}}^{2}\right)$.

Though it makes $c_{ \pm}$simple to compute, this dispersion relation overestimates the maximum characteristic speeds by a factor $\leqslant 2$, which has the net effect of making the code more dissipative. Though additional dissipation may smear important physical features in our GRMHD flows, it also acts to help stabilize evolutions. Note that this approximate dispersion relation is widely used in multiple codes within the GRMHD community (e.g., WhiskyMHD [39], GRHydro [50], HARM3D [53]).

Once the maximum and minimum speeds $c_{ \pm}$have been computed at left and right faces, the standard Harten-Lax-van Leer (HLL), approximate Riemann solver [42] is then applied to compute fluxes for the three conservative variables $\boldsymbol{U}=\left\{\rho_{*}, \tilde{\tau}, \tilde{S}_{i}\right\}$ :

$$
F^{\mathrm{HLL}}=\frac{c^{-} F_{\mathrm{R}}+c^{+} F_{\mathrm{L}}-c^{+} c^{-}\left(U_{\mathrm{R}}-U_{\mathrm{L}}\right)}{c^{+}+c^{-}}
$$

where $c^{ \pm}= \pm \max \left(0, c_{ \pm}^{\mathrm{R}}, c_{ \pm}^{\mathrm{L}}\right)$, and $U_{\mathrm{R}, \mathrm{L}}$ are the conservative variables $\boldsymbol{U}$ computed from the right and left reconstructed primitive values $\boldsymbol{P}_{\mathrm{R}, \mathrm{L}}$, respectively.

Upon computing the HLL flux at cell interfaces, the final step in evaluating the flux terms in the evolution equations of $\rho_{*}, \tilde{S}_{i}$, and $\tilde{\tau}$ (equation (18)) is to differentiate the computed HLL flux terms along the same direction in which they were evaluated. After computing the HLL flux in the $x$-direction we calculate the $x$-derivative of the flux as:

$$
\left(\partial_{x} F^{x}\right)_{i, j, k}=\frac{F_{\mathrm{HLL} i+\frac{1}{2}, j, k}^{x}-F_{\mathrm{HLL} i-\frac{1}{2}, j, k}^{x}}{\Delta x} .
$$

The remaining $y$ - and $z$-terms in the $\partial_{m} F^{m}$ sum are added to the sum as reconstruction proceeds along the $y$ - and $z$-directions, respectively. 
Table 1. Storage location on grid of the magnetic field $B^{i}$ and vector potential $\mathcal{A}_{\mu}$. Note that $\boldsymbol{P}$ is the vector of primitive variables $\left\{\rho_{0}, P, v^{i}\right\}$.

\begin{tabular}{lc}
\hline Variable(s) & Storage location \\
\hline Metric terms, $\boldsymbol{P}, \rho_{*}, \tilde{S}_{i}, \tilde{\tau}$ & $(i, j, k)$ \\
$B^{x}, \tilde{B}^{x}$ & $\left(i+\frac{1}{2}, j, k\right)$ \\
$B^{y}, \tilde{B}^{y}$ & $\left(i, j+\frac{1}{2}, k\right)$ \\
$B^{z}, \tilde{B}^{z}$ & $\left(i, j, k+\frac{1}{2}\right)$ \\
$A_{x}$ & $\left(i, j+\frac{1}{2}, k+\frac{1}{2}\right)$ \\
$A_{y}$ & $\left(i+\frac{1}{2}, j, k+\frac{1}{2}\right)$ \\
$A_{z}$ & $\left(i+\frac{1}{2}, j+\frac{1}{2}, k\right)$ \\
$\sqrt{\gamma} \Phi$ & $\left(i+\frac{1}{2}, j+\frac{1}{2}, k+\frac{1}{2}\right)$ \\
\hline
\end{tabular}

As the source terms of equation (18) have already been computed, to complete the evaluation of $\partial_{t} \rho_{*}, \partial_{t} \tilde{S}_{i}$, and $\partial_{t} \tilde{\tau}$, all components of the $\partial_{m} F^{m}$ sum are then subtracted from the source terms. These data are then passed to the MoL thorn, which is capable of managing a number of explicit time-stepping techniques. Although MoL supports a total-variation diminishing third-order Runge-Kutta time integrator, we typically choose the RK4 scheme for all evolution variables, both in IllinoisGRMHD and the chosen spacetime evolution thorn, as we find that RK4 minimizes the total error when evolving both the spacetime and the fluid.

In parallel with evaluating the flux and source terms for $\rho_{*}, \tilde{S}_{i}$, and $\tilde{\tau}$, IllinoisGRMHD employs a vector-potential-based constrained transport scheme to evolve the magnetic fields, which is detailed in the next section.

\subsection{Vector-potential-based constrained transport scheme}

Constrained-transport schemes maintain $\nabla \cdot \boldsymbol{B}=0$ through careful finite differencing of the magnetic induction equation flux terms (equation (12)). Such schemes have proven highly robust in the context of strongly curved spacetimes; in particular those inhabited by at least one BH. These schemes are most commonly and most directly applied in the context of uniformresolution grids. However, their use with AMR grids can be complicated, as maintaining the divergenceless constraint at refinement boundaries requires that special interpolations be performed during prolongation/restriction. Such prolongation/restriction operators have been devised $[9,10]$, but must be fine-tuned to the particular AMR implementation.

IllinoisGRMHD applies an alternative constrained-transport scheme, introduced by [20]. In this scheme, the magnetic induction equation (12) is recast as an evolution equation for the magnetic vector potential (equation (16)). This scheme has two important advantages. First, it produces identical output to the standard, staggered constrained-transport scheme on uniform resolution grids and thus shares its robustness. Second, evolving the vector potential enables us to use any interpolation scheme at AMR refinement boundaries without introducing nonzero divergence to the magnetic fields, so long as we compute $B^{i}$ from the interpolated $A_{i}$.

The remainder of this section details the staggered constrained-transport scheme adopted within IllinoisGRMHD. First, we define the staggerings of individual gridfunctions and the 
computation of $B^{m}$ from $A_{m}$. Then the technique of reconstruction (equation (12)) on staggered cell faces is outlined, and finally the Riemann solver is described.

Computation of $B^{m}$ from $A_{m}$ : in employing the standard, staggered constrained transport scheme, magnetic fields are defined at gridpoints that are staggered with respect to other conservative variables, as specified in table 1 . Notice that $A_{m}$ is staggered so that $B^{m}$ may be computed immediately from equation (15) using the following finite difference representation, accurate to second order:

$$
B_{i+\frac{1}{2}, j, k}^{x}=\sqrt{\gamma}_{i+\frac{1}{2}, j, k}\left(\partial_{y} A_{z}\right)^{i+\frac{1}{2}, j, k}-\sqrt{\gamma}_{i+\frac{1}{2}, j, k}\left(\partial_{z} A_{y}\right)^{i+\frac{1}{2}, j, k},
$$

where

$$
\begin{aligned}
& \sqrt{\gamma}_{i+\frac{1}{2}, j, k}=\exp \left(6 \times \frac{1}{2}\left[\phi_{i, j, k}+\phi_{i+1, j, k}\right]\right), \\
& \left(\partial_{y} A_{z}\right)^{i+\frac{1}{2}, j, k}=\frac{A_{z}^{i+\frac{1}{2}, j+\frac{1}{2}, k}-A_{z}^{i+\frac{1}{2}, j-\frac{1}{2}, k}}{\Delta y}, \text { and } \\
& \left(\partial_{z} A_{y}\right)^{i+\frac{1}{2}, j, k}=\frac{A_{y}^{i+\frac{1}{2}, j, k+\frac{1}{2}}-A_{y}^{i+\frac{1}{2}, j, k-\frac{1}{2}}}{\Delta z} .
\end{aligned}
$$

Here, $\phi=(1 / 12) \log \gamma$ is the BSSN conformal exponent. Using equation (27) as a template, $B^{y}$ and $B^{z}$ can be written via straightforward permutation of vector indices $\{x, y, z\}$, accounting for the appropriate staggerings. Our finite differencing scheme is specified so that the divergence of a curl is identically zero to roundoff error. In this way, $B^{m}$ is guaranteed to be divergenceless at all but the outermost ghost-zones on any given refinement level, so long as $A_{m}$ is computed at all points. As with the other conservative variables, reconstruction of the flux terms for $A_{m}$ requires three ghostzones (as discussed in the next section), so the prerequisite step of computing $B^{m}$ from $A_{m}$ adds an additional ghostzone, bringing the total number to four. However, we have found that application of a copy boundary condition on $B^{m}$ to the outermost gridpoint on each refinement level, coupled to the use of only three ghostzones, results in qualitatively identical results to runs that use four ghostzones. We find this to be the case even in the most stringent tests, such as a magnetized BH accretion disk crossing multiple refinement boundaries, as in [36, 40, 41]. Thus by default, we have used three ghostzones in all GRMHD simulations.

Flux reconstruction of the induction equation: accounting for staggerings, the evolution equation for $A_{z}$ (dropping the EM gauge terms to focus on the flux term of the induction equation) is given by

$$
\partial_{t} A_{z}^{i+\frac{1}{2} j+\frac{1}{2}, k}=-\mathcal{E}_{i+\frac{1}{2}, j+\frac{1}{2}, k}^{z}
$$

where

$$
\mathcal{E}^{z}=-v^{x} \tilde{B}^{y}+v^{y} \tilde{B}^{x}
$$

is the flux term in the standard magnetic induction equation (12). Following [20], we compute this flux term to staggered cell faces for $A_{z}$ and then evaluate the HLL flux generalized for staggered grids. As $\mathcal{E}^{z}$ does not appear within a derivative of the $A_{z}$ induction equation (as it does in the $\tilde{B}^{z}$ induction equation), the flux is not directly finite-differenced prior to passing the rhs of $\partial_{t} A_{z}$ to the time-stepping routines. Instead, the spatial finite difference is computed 
after each RK4 iteration when $B^{m}$ is computed from $A_{m}$. Critically, the order in which spatial and temporal derivatives are evaluated is the only difference between the standard, staggered constrained-transport scheme and our vector-potential based staggered constrainedtransport scheme. And since spatial and temporal derivative operators commute within IllinoisGRMHD's current framework, both schemes are identical on uniform meshes.

Returning to the evaluation of $\mathcal{E}^{z}$, recall that primitives such as $v^{i}$ are defined at grid points $(i, j, k)$, so computing the value $\mathcal{E}^{z}$ at $\left(i+\frac{1}{2}, j+\frac{1}{2}, k\right)$ requires two successive onedimensional reconstructions of $v^{x}$ and $v^{y}$ : first in the $x$ or $y$-direction and then in the $y$ - or $x$-direction, respectively. $\tilde{B}^{x}$ and $\tilde{B}^{y}$ already exist on staggered gridpoints (see table 1 ), requiring only a single reconstruction in the $y$ - and $x$-direction, respectively. Reconstruction is handled via the same PPM scheme as described in section 3.1.

Approximate Riemann solver for $A_{i}$ : the standard HLL formula (25) for $\mathcal{E}^{z}$, generalized to the appropriate staggered gridfunctions, is given by:

$$
\begin{aligned}
\left(\mathcal{E}^{z}\right)^{\mathrm{HLL}}= & \frac{c_{x}^{+} c_{y}^{+} \mathcal{E}_{\mathrm{LL}}^{z}+c_{x}^{+} c_{y}^{-} \mathcal{E}_{\mathrm{LR}}^{z}+c_{x}^{-} c_{y}^{-} \mathcal{E}_{\mathrm{RL}}^{z}+c_{x}^{-} c_{y}^{-} \mathcal{E}_{\mathrm{RR}}^{z}}{\left(c_{x}^{+}+c_{x}^{-}\right)\left(c_{y}^{+}+c_{y}^{-}\right)} \\
& +\frac{c_{x}^{+} c_{x}^{-}}{c_{x}^{+}+c_{x}^{-}}\left(\tilde{B}_{\mathrm{R}}^{y}-\tilde{B}_{\mathrm{L}}^{y}\right)-\frac{c_{y}^{+} c_{y}^{-}}{c_{y}^{+}+c_{y}^{-}}\left(\tilde{B}_{\mathrm{R}}^{x}-\tilde{B}_{\mathrm{L}}^{x}\right) .
\end{aligned}
$$

In the above formula, $\mathcal{E}_{\mathrm{LR}}^{z}$ denotes the reconstructed left state in the $x$-direction and right state in the $y$-direction. Other symbols involving $\mathcal{E}^{z}$ are interpreted in the similar fashion. $\tilde{B}_{\mathrm{R}}^{y}\left(\tilde{B}_{\mathrm{L}}^{y}\right)$ denotes the reconstructed right (left) state of $\tilde{B}^{y}$ in the $x$-direction, and $\tilde{B}_{\mathrm{R}}^{x}\left(\tilde{B}_{\mathrm{L}}^{x}\right)$ denotes the reconstructed right (left) state in the $y$-direction. The $c_{x}^{ \pm}$and $c_{y}^{ \pm}$should be computed by taking the maximum characteristic speed among the four reconstructed states. However, we set them equal to the maximum over the two neighboring interface values for simplicity, as suggested in [20], using the technique described in section 3.1 to estimate the speeds. The formula for $\left(\mathcal{E}^{x}\right)^{\mathrm{HLL}}$ is obtained from equation (33) by permuting the indices $z \rightarrow x, x \rightarrow y$ and $y \rightarrow z$, whereas the formula for $\left(\mathcal{E}^{y}\right)^{\mathrm{HLL}}$ is obtained from equation (33) by permuting the indices $z \rightarrow y, x \rightarrow z$ and $y \rightarrow x$.

\subsection{Evolution of the densitized EM scalar potential $[\sqrt{\gamma} \Phi]$}

Incorporating the staggering of the EM gauge variable $[\sqrt{\gamma} \Phi]$ (as specified in table 1 ), the evolution equation for $[\sqrt{\gamma} \Phi]$ (equation (17)) may be written:

$\partial_{t}[\sqrt{\gamma} \Phi]_{i+\frac{1}{2}, j+\frac{1}{2}, k+\frac{1}{2}}=-\underbrace{\partial_{m}\left(\alpha \psi^{2} \tilde{\gamma}^{m n} A_{n}\right)}_{\text {Term (1) }}+\underbrace{\partial_{m}\left(\beta^{m}[\sqrt{\gamma} \Phi]\right)}_{\text {Term (2) }}-\underbrace{\xi \alpha[\sqrt{\gamma} \Phi]}_{\text {Term (3) }}$,

where $\psi$ is the standard BSSN conformal factor and the relations $\gamma^{m n}=\psi^{-4} \tilde{\gamma}^{m n}$ and $\sqrt{\gamma}=\psi^{6}$ have been applied. The left-hand side of the equation is evaluated at $\left(i+\frac{1}{2}, j+\frac{1}{2}, k+\frac{1}{2}\right)$, yet $A_{m},[\sqrt{\gamma} \Phi]$, and metric quantities on the rhs of this equation all possess different staggerings. Thus special care must be taken so that the derivatives on the rhs of this equation are evaluated at gridpoints $\left(i+\frac{1}{2}, j+\frac{1}{2}, k+\frac{1}{2}\right)$. To accomplish this, quantities within the rhs derivatives are first interpolated to consistent points prior to evaluation of the derivatives. Note that this strategy differs from the evolution of other GRMHD variables, in that no reconstruction is applied. Methods for computing these terms on the rhs are as follows: 
$\operatorname{Term}(1)$ : for the $x$-derivative, all quantities within the derivative operator $\left(\left[\alpha \psi^{2}\right] \tilde{\gamma}^{m x} A_{m}\right)$ are first interpolated to $\left(i, j+\frac{1}{2}, k+\frac{1}{2}\right)$. At second-order accuracy, interpolations to staggered gridpoints are trivial, requiring only averages of neighboring unstaggered points. For example, interpolation of $\alpha \psi^{2}$ from $(i, j, k)$ to $\left(i, j+\frac{1}{2}, k+\frac{1}{2}\right)$ is performed in two steps:

(i) $\left[\alpha \psi^{2}\right]_{i, j+\frac{1}{2}, k}=\frac{1}{2}\left(\left[\alpha \psi^{2}\right]_{i, j, k}+\left[\alpha \psi^{2}\right]_{i, j+1, k}\right)$

(ii) $\left[\alpha \psi^{2}\right]_{i, j+\frac{1}{2}, k+\frac{1}{2}}=\frac{1}{2}\left(\left[\alpha \psi^{2}\right]_{i, j+\frac{1}{2}, k}+\left[\alpha \psi^{2}\right]_{i, j+\frac{1}{2}, k+1}\right)$.

Once $\left[\alpha \psi^{2}\right], \tilde{\gamma}^{m x}, A_{y}$, and $A_{z}$ have been interpolated in this way to $\left(i, j+\frac{1}{2}, k+\frac{1}{2}\right)$, the derivative $\partial_{x}\left(\left[\alpha \psi^{2}\right] \tilde{\gamma}^{x m} A_{m}\right)$ is computed to second order as follows

$\partial_{x}\left(\left[\alpha \psi^{2}\right] \tilde{\gamma}^{x m} A_{m}\right)_{i+\frac{1}{2}, j+\frac{1}{2}, k+\frac{1}{2}}=\frac{\left(\left[\alpha \psi^{2}\right] \tilde{\gamma}^{x m} A_{m}\right)_{i+1, j+\frac{1}{2}, k+\frac{1}{2}}-\left(\left[\alpha \psi^{2}\right] \tilde{\gamma}^{x m} A_{m}\right)_{i, j+\frac{1}{2}, k+\frac{1}{2}}}{\Delta x}$.

Other derivatives in the sum $\partial_{m}\left(\alpha \psi^{2} \tilde{\gamma}^{m n} A_{n}\right)$ are computed in the same fashion.

Term (2): the computation of this term is made easier by the fact that $[\sqrt{\gamma} \Phi]$ is staggered at $\left(i+\frac{1}{2}, j+\frac{1}{2}, k+\frac{1}{2}\right)$ already. So to evaluate the derivative, $\beta^{m}$ is first interpolated from $(i, j, k)$ to $\left(i+\frac{1}{2}, j+\frac{1}{2}, k+\frac{1}{2}\right)$ using the same interpolation strategy as with Term (1). Next, notice that this term is basically a shift advection term on the EM gauge quantity $[\sqrt{\gamma} \Phi]$. Such advection terms are typically upwinded within the metric evolution thorn, so for consistency we apply the same upwinding strategy when evaluating this derivative:

$$
\partial_{m}\left(\beta^{m}[\sqrt{\gamma} \Phi]\right)= \begin{cases}D_{m}^{-}\left(\beta^{m}[\sqrt{\gamma} \Phi]\right) & \text { if } \beta^{m}<0, \\ D_{m}^{+}\left(\beta^{m}[\sqrt{\gamma} \Phi]\right) & \text { otherwise }\end{cases}
$$

where the second-order operators are

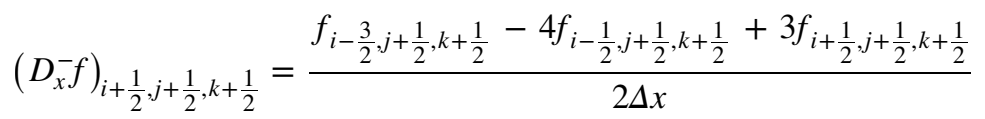

and

$$
\left(D_{x}^{+} f\right)_{i+\frac{1}{2}, j+\frac{1}{2}, k+\frac{1}{2}}=\frac{-f_{i+\frac{5}{2}, j+\frac{1}{2}, k+\frac{1}{2}}+4 f_{i+\frac{3}{2}, j+\frac{1}{2}, k+\frac{1}{2}}-3 f_{i+\frac{1}{2}, j+\frac{1}{2}, k+\frac{1}{2}}}{2 \Delta x}
$$

for the derivative in the $x$-direction. Derivatives in the $y$ - and $z$-directions follow in a straightforward fashion.

Term (3): the computation of this term is also made easier by the fact that $[\sqrt{\gamma} \Phi]$ is staggered at $\left(i+\frac{1}{2}, j+\frac{1}{2}, k+\frac{1}{2}\right)$ already. So to evaluate it, only $\alpha$ must be interpolated 
from $(i, j, k)$ to $\left(i+\frac{1}{2}, j+\frac{1}{2}, k+\frac{1}{2}\right)$ using the same interpolation strategy as with Term (1).

\subsection{Conservatives-to-primitives solver}

After the conservative GRMHD variables have been updated at all gridpoints, with boundary conditions and prolongation/restriction operators applied, the primitive variables must then be computed from the conservative variables. This is not a trivial endeavor, as the conservative variables generally depend on the primitive variables in a nonlinear way, requiring the implementation of a root-finding method. To this end, IllinoisGRMHD employs the twodimensional (2D) Newton-Raphson solver of [52, 53].

Truncation errors originating from spatial and temporal finite differencing, as well as interpolation, prolongation, and restriction operations can push the evolved GRMHD quantities to unphysical values, resulting in either unphysical values for the primitive variables or no values at all. For definitions of unphysical values of the GRMHD quantities please see the appendix A of [28]. So prior to calling the 2D Newton-Raphson solver, we perform a number of checks that determine whether the conservative variables are in a physically valid range. If they are not, they are modified prior to calling the root-finder. Even with these checks, the Newton-Raphson solver will occasionally fail to find a root. This is very rare, and almost always occurs in a low-density atmosphere or inside a BH. In such an instance, we set the pressure to $P_{\text {cold }}$, which guarantees a successful inversion. The implementation of these checks and modifications have been described in detail in appendix A of [28].

After the Newton-Raphson solver has successfully found a set of primitives, the primitives are checked for physicality, and if they are not in the physical range, they are minimally modified until they return to the physical range. First, if the velocity is found to be superluminal, the speed is reduced to IllinoisGRMHD's default Lorentz factor limit, which is set to $W=10$, where $W$ is the Lorentz factor of the fluid as measured by a normal observer. Next, IllinoisGRMHD does not include any cooling mechanism, which means that for evolutions adopting a $\Gamma$-law equations of state, the pressure should not physically drop below $P_{\text {cold }}$. So a pressure floor of $0.9 P_{\text {cold }}$ is imposed. Increasing this floor to $P_{\text {cold }}$ exactly results in large central density drifts in TOV star evolutions. Simulations can crash in the other extreme, if $P / P_{\text {cold }}$ becomes too large. This typically only happens in very low density regions or inside BHs. So at densities $\rho_{0}<100 \rho_{\text {atm }}$ or deep inside $\mathrm{BH}$ horizons, a ceiling on $P$ of $100 P_{\text {cold }}$ is enforced (see appendix A of [28] for more details).

\subsection{Outer boundary conditions for $A_{i},[\sqrt{\gamma} \Phi], P, \rho_{0}$, and $v^{i}$}

Updating evolved variables within IllinoisGRMHD requires three ghostzones per RK4 iteration, and at the end of each iteration, outer boundary conditions are applied to $A_{i},[\sqrt{\gamma} \Phi]$, $P, \rho_{0}$, and $v^{i}$ to fill these ghostzones. The algorithm applies the outer boundary conditions in all directions, from the innermost gridpoint outward, as follows. For example, in the positive $x$-direction, the first outer gridpoint $i+1$ is defined as 


$$
\boldsymbol{E}_{i+1}= \begin{cases}\boldsymbol{E}_{i}, & \text { if } \boldsymbol{E} \in\left\{P, \rho_{0}, v^{y}, v^{z}\right\}, \text { or } \boldsymbol{E} \equiv v^{x} \text { and } v^{x} \geqslant 0, \\ 0, & \text { if } \boldsymbol{E} \equiv v^{x}, \text { and } v^{x}<0, \\ 2 \boldsymbol{E}_{i}-\boldsymbol{E}_{i-1}, & \text { if } \boldsymbol{E} \in\left\{[\sqrt{\gamma} \Phi], A_{x}, A_{y}, A_{z}\right\} .\end{cases}
$$

And for the negative $x$-direction, the first outer gridpoint $i-1$ is defined as

$$
\boldsymbol{E}_{i-1}= \begin{cases}\boldsymbol{E}_{i}, & \text { if } \boldsymbol{E} \in\left\{P, \rho_{0}, v^{y}, v^{z}\right\}, \text { or } \boldsymbol{E} \equiv v^{x} \text { and } v^{x} \leqslant 0, \\ 0, & \text { if } \boldsymbol{E} \equiv v^{x}, \text { and } v^{x}>0, \\ 2 \boldsymbol{E}_{i}-\boldsymbol{E}_{i+1}, & \text { if } \boldsymbol{E} \in\left\{[\sqrt{\gamma} \Phi], A_{x}, A_{y}, A_{z}\right\} .\end{cases}
$$

As for the positive/negative $y$ and $z$ directions, the procedure is the same, replacing $v^{x} \leftrightarrow v^{y}$ and $v^{x} \leftrightarrow v^{z}$, respectively.

In this way, linear extrapolation outer boundary conditions are applied to the vector potential variables $\left\{[\sqrt{\gamma} \Phi], A_{i}\right\}$ and zero-derivative, outflow outer boundary conditions are applied to the hydrodynamic variables $\left\{P, \rho_{0}, v^{i}\right\}$. These conditions are applied to the innermost gridpoints on the three-gridpoint-thick outer boundary surface, first in the positive $x$, then $y$, then $z$ directions, followed by the negative $x$, then $y$, then $z$ directions. Next, they are applied to the second innermost gridpoints on the outer boundary surface in all directions, and finally to the outermost point.

In addition to full 3D Cartesian grids with no symmetries assumed, IllinoisGRMHD also supports the application of a symmetry condition across the $x y$-plane. In this case the negative $z$-direction outer boundary condition is not applied, letting the Cactus/Carpet parallel AMR infrastructure impose the reflection symmetry.

\subsection{Linkage of IllinoisGRMHD to the rest of the ET}

In order to evolve the GRMHD equations in a dynamical spacetime context, IllinoisGRMHD must be coupled to a separate module that evolves the spacetime metric, typically using components of the stress-energy tensor produced by IllinoisGRMHD as source terms. The ET is based within the Cactus infrastructure, thus modules are called 'thorns', of which IllinoisGRMHD is one. ET is structured so that thorns evaluating the spacetime metric evolution equations (i.e., the left-hand side of Einstein's equations) must couple to a common interface thorn, called ADMBase. Similarly, thorns that evaluate evolution equations governing the rhs of Einstein's equations couple to an interface thorn called TmunuBase. TmunuBase and ADMBase are designed to interface seamlessly, so that GRMHD evolution thorns coupled to TmunuBase will automatically work with any spacetime evolution thorn properly coupled to ADMBase. Thus, since IllinoisGRMHD is fully coupled to TmunuBase, it is immediately compatible with all spacetime evolution formulations within ET, including BSSN, and conformal and covariant Z4 [1] (both provided by the McLachlan [15] Thorn ${ }^{9}$ ). For dynamical spacetime evolutions within this paper, IllinoisGRMHD is coupled to the McLachlan BSSN thorn.

9 Kranc assembles numerical code, http://kranccode.org. 


\section{Code validation tests}

This section compares the results of IllinoisGRMHD to those of two other codes written using the ET infrastructure: GRHydro, which is the only other open-source GRMHD code within ET, and the original, closed-source GRMHD code of the Illinois group (OrigGRMHD), on which IllinoisGRMHD is based. OrigGRMHD has been subjected to a large battery of stringent test-bed problems, including but not limited to standard 1D relativistic MHD shock tests, 2D cylindrical blast explosion tests, magnetized Bondi accretion and stellar collapse tests as well as (self-)convergence tests [30]. Though it may be argued that IllinoisGRMHD has not been as robustly tested as GRHydro or OrigGRMHD, we demonstrate here that IllinoisGRMHD and OrigGRMHD results agree to roundoff error, indicating that both are algorithmically identical, and GRHydro and IllinoisGRMHD results agree within truncation error, indicating that both can be expected to converge to the same result.

To confirm that two codes generate results that agree to roundoff error, we measure the number of significant digits of agreement between codes, monitoring one quantity of interest. Given that the equations solved are highly nonlinear, roundoff-error violations in one quantity will spread to all others, so we generally focus on one quantity of interest. In the following sections, we monitor either the central density $\rho_{c}$ of a NS over time, or the integral of the restmass density $\rho_{b}$ along the $x$-axis. We perform runs with different codes and compute the number of significant digits of agreement between a pair of codes, $s$, at a given time $t$, via the equation:

$$
s(t)=\log _{10}\left|\frac{\rho_{c, 1}(t)-\rho_{c, 2}(t)}{\rho_{c, 1}(t)}\right|,
$$

where the subscript ' 1 ' denotes the unperturbed run with OrigGRMHD, and the subscript ' 2 ' denotes either the perturbed initial data run with OrigGRMHD or an unperturbed run with IllinoisGRMHD.

The first two parts of this section (sections 4.1 and 4.2) demonstrate that IllinoisGRMHD and OrigGRMHD generate output identical to roundoff error, in two complementary, highly challenging tests. In the first test (section 4.1), a weakly magnetized TOV star is evolved over many dynamical timescales and grid light-crossing times. The second test (section 4.2) exposes both codes to a type of 'fuzzing', in which random initial data are evolved. Unlike the first test, initial data in the second test contain strong shocks, highly magnetized and highly relativistic, stochastic flows, as well as nontrivial, discontinuous spacetime-metric and extrinsic curvature components. Despite the harshness of the second test, both codes are shown in section 4.2 to produce roundoff-error identical results over many grid light-crossing times. Results from these tests are highly significant, as they demonstrate that IllinoisGRMHD yields identical results to the 'battle-hardened', trusted OrigGRMHD code. We conclude that IllinoisGRMHD must therefore pass any code validation test that OrigGRMHD has passed, and can be used as a drop-in replacement for OrigGRMHD.

In our final validation test, both IllinoisGRMHD and GRHydro evolve unmagnetized, stable TOV stars in a dynamical spacetime backdrop, and are shown to converge to the same result at the expected order, though IllinoisGRMHD exhibits slightly slower central density drift and lower Hamiltonian constraint violations at a given resolution. 


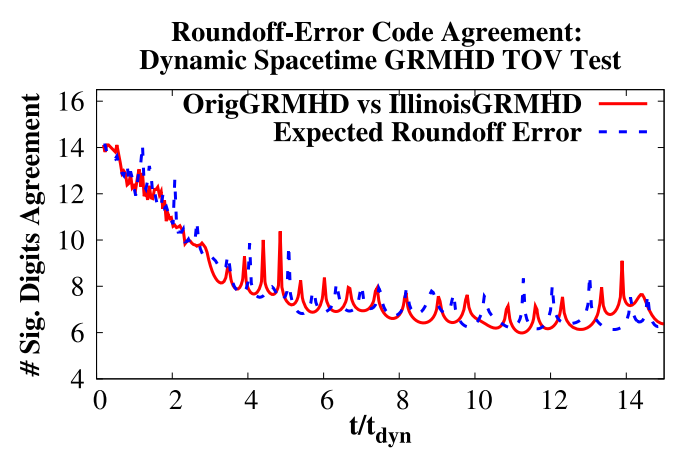

Figure 1. Significant digits of agreement between pairs of codes, monitoring the central density of a magnetized neutron star in a dynamic-spacetime GRMHD simulation versus time, as measured in dynamical timescales $t_{\mathrm{dyn}}=1 / \sqrt{\rho_{0, \max }}$. The solid red line shows the number of significant digits of agreement between IllinoisGRMHD and OrigGRMHD. The dashed blue line shows the expected roundoff error, measured as the number of significant digits of agreement between OrigGRMHD and itself with a 15th-significant-digit perturbation to the initial density of the magnetized neutron star. This run was performed on 8 parallel processes on a desktop computer. All details from this simulation are provided in appendix A, and equation (41) is applied to measure the number of significant digits.

\subsection{IllinoisGRMHD and OrigGRMHD: roundoff-error agreement in evolving magnetized TOV star}

IllinoisGRMHD, OrigGRMHD, and GRHydro all implement double-precision, 64 bit floating point arithmetic, which represents numbers to between 15 and 17 significant digits. Given the sophisticated and iterative nature of these GRMHD codes, initial machine-precision differences can grow enormously over time. As an example, we multiply the initial rest-mass density of a weakly magnetized TOV star by $1+10^{-15}$, yielding a 15 th significant digit perturbation. We then perform the evolution, measuring the number of significant digits of agreement between this perturbed run and an unperturbed evolution, through 15 dynamical timescales and on AMR grids with multiple levels of refinement. The dashed blue line of figure 1 plots the result from this test. Notice that the number of significant digits of agreement quickly drops from 14 digits, plateauing to between 6 and 8 digits of agreement.

We were careful to develop IllinoisGRMHD so that its results agree with OrigGRMHD to roundoff error, and figure 1 confirms that for this weakly magnetized TOV star test, the number of significant digits of agreement between IllinoisGRMHD and OrigGRMHD (solid red line) follow the same curve as the expected roundoff error intrinsic to OrigGRMHD (dashed blue line). For full details of the physical scenario modeled here, as well as the grid parameters, see appendix A.

\subsection{IllinoisGRMHD and OrigGRMHD: roundoff-error agreement in evolving random initial data}

Although we have demonstrated that when evolving weakly magnetized TOV stars on AMR grids, IllinoisGRMHD and OrigGRMHD produce results that agree to roundoff error, one might argue that even though there is a sharp discontinuity at the stellar surface, this code test is insufficient for truly demonstrating roundoff-level agreement, as it lacks strong shocks and highly relativistic, highly magnetized fluid flows. To address this potential criticism, we developed a random initial data module that sets up both weak and strong stochastic GRMHD flows atop an artificially-static, weak and strong-field stochastic spacetime background that is 


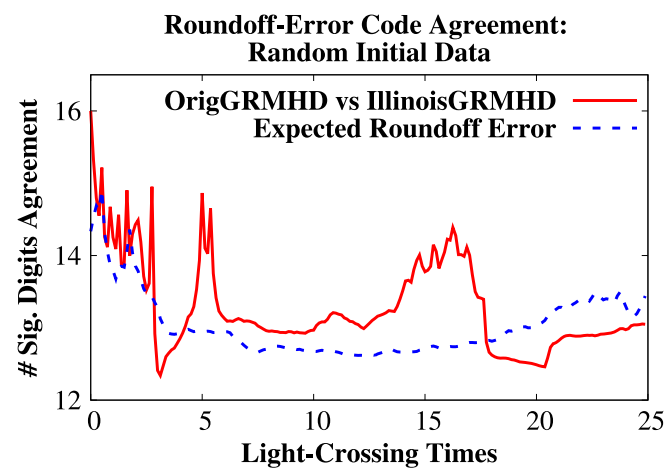

Figure 2. Significant digits of agreement between pairs of codes, monitoring the restmass density $\rho_{0}$ summed along the $x$-axis on the finest AMR level. The solid red line shows the number of significant digits of agreement between IllinoisGRMHD and OrigGRMHD. The dashed blue line shows the expected roundoff error, measured as the number of significant digits of agreement between OrigGRMHD and itself with a random 15th-significant-digit perturbation to all primitive GRMHD variables. This run was performed on 8 parallel processes on a desktop computer, and was shown to agree with the single-process OrigGRMHD run to roundoff-error as well. All details from this simulation are provided in appendix B, and equation (41) is applied to measure the number of significant digits. Unlike figure 1, where the dashed (blue) curve matches the variability of the solid (red) curve, here the dashed (blue) curve exhibits significantly less variability than the solid (red) curve. We find this apparent inconsistency disappears when we substitute the integrated value of the density along the $x$-axis with the maximum value along the axis. Thus the discrepancy in behavior appears to be due to the choice of quantity adopted for this comparison. Regardless of the quantity used to measure the differences, the results demonstrate that the two codes agree to roundoff error.

nearly conformally flat. The stochastic nature of these data means that both metric and GRMHD quantities suffer from both weak and strong discontinuities from one spatial point to the next, providing a robust test of the HRSC algorithms within these GRMHD codes, as well as a confirmation of the stability of both GRMHD codes to fuzz testing. We stress that although the chosen metric has Lorentzian signature and the spatial three-metric is positivedefinite, these initial data are for numerical convenience only and are not designed to satisfy Einstein's equations. In fact, when these GRMHD data are evolved forward in time, spacetime field variables are strictly held fixed in time.

All components of spacetime and GRMHD tensors and vectors are nonzero, randomly fluctuating from one spatial point to the next, with each component having a unique magnitude. As a result, this module has been useful in checking for typos in the GRMHD evolution equations, which were completely rewritten in IllinoisGRMHD. For example, if by mistake $\gamma_{x y}$ were written $\gamma_{x z}$ in any of the GRMHD equations, then because these components have differing magnitudes, IllinoisGRMHD and the original GRMHD code of the Illinois group would not agree to roundoff precision and the test would fail. This module was used extensively in the first stages of IllinoisGRMHD development to find such typos, as well as truncation-error-level algorithmic differences between the old and new codes. All such typos and algorithmic differences were fixed and modified, respectively, so that roundoff-level agreement could be demonstrated. A full description of the random initial data module and grid setup is provided in appendix B.

As with the magnetized TOV roundoff-error test of section 4.1, we measure the expected level of roundoff error by first adding a random, 15th-digit perturbation to all GRMHD 
primitive variables after they are set. Then we evolve both perturbed and unperturbed initial data with the trusted OrigGRMHD code. The difference grows with time, but plateaus to about 13 digits over time, as shown in figure 2.

Next, the same unperturbed initial data are evolved on the same grids with IllinoisGRMHD, and the results confirm that IllinoisGRMHD and OrigGRMHD indeed agree to within expected roundoff error, through at least 25 light-crossing times. All of these runs were performed on 8 parallel processes.

\subsection{IllinoisGRMHD and GRHydro: unmagnetized TOV star convergence tests}

The open-source IllinoisGRMHD and closed-source OrigGRMHD have been shown to produce roundoff-error identical results even when evolving very harsh, relativistic, strongly magnetized, discontinuous initial data. IllinoisGRMHD represents the second open-source, dynamical spacetime GRMHD module, the first being GRHydro [50]. Both are based in the ET, which provides a particularly convenient infrastructure for performing GRMHD simulations in a dynamical spacetime context. GRHydro contains a large number of features, including a variety of reconstruction options, approximate Riemann solvers, and outer boundary options. OrigGRMHD contains many such features as well, but nearly all of these features are not robust in the context of $\mathrm{BH}$-inhabited spacetimes and have thus remained unused for years. IllinoisGRMHD contains only the features from OrigGRMHD that have been used in all recent papers by the Illinois NR group (e.g., [28, 33, 36, 40, 41, 59]).

This section compares results between IllinoisGRMHD and the standard, FORTRAN version of GRHydro, using identical initial data, computational grids, dynamical spacetime evolution (BSSN) modules, reconstruction scheme, and Riemann solver. Since IllinoisGRMHD has been shown to agree with OrigGRMHD to roundoff precision, these tests can also be seen as a proxy comparison between GRHydro and OrigGRMHD.

As detailed in appendix C, both IllinoisGRMHD and GRHydro evolve the same physical scenario in this test as in section 4.1, but with the magnetic fields inside the TOV star set to zero. GRHydro options were chosen so that its algorithms would be identical to IllinoisGRMHD. Despite the basic algorithms being the same, both codes differ significantly in how they are implemented. This difference in implementation should result in truncationlevel differences between the two codes, but instead we find slightly different convergence properties between the codes.

A quantity $Q(\Delta x)$ that converges to zero at $n$th order with increasing resolution (i.e., decreasing grid spacing $\Delta x)$ satisfies

$$
\frac{Q\left(\Delta x_{1}\right)}{Q\left(\Delta x_{2}\right)}=\left(\frac{\Delta x_{1}}{\Delta x_{2}}\right)^{n} \text {. }
$$

Thus the convergence order to zero, $n$, is written as follows:

$$
n=\log \left(\frac{Q\left(\Delta x_{1}\right)}{Q\left(\Delta x_{2}\right)}\right) / \log \left(\frac{\Delta x_{1}}{\Delta x_{2}}\right) .
$$

Figure 3 demonstrates that for this stable, equilibrium TOV star, truncation errors lead to nonzero drifts in star's central density and the L2-Norm of the Hamiltonian constraint, which each converge to zero at roughly second-order $(n(t) \approx 2$, where $n$ is as defined in equation (43)). Notice that L2-Norm Hamiltonian constraint convergence order fluctuates significantly in GRHydro evolutions, as compared to IllinoisGRMHD. Additionally, at the highest resolution chosen (resolving the NS diameter to approximately 80 gridpoints), 


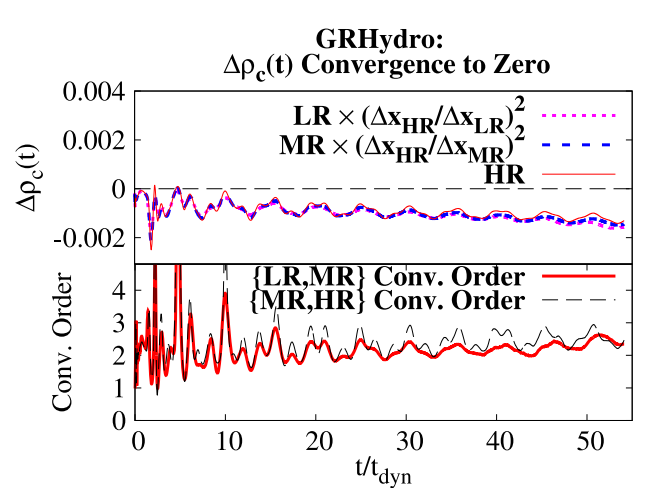

GRHydro:

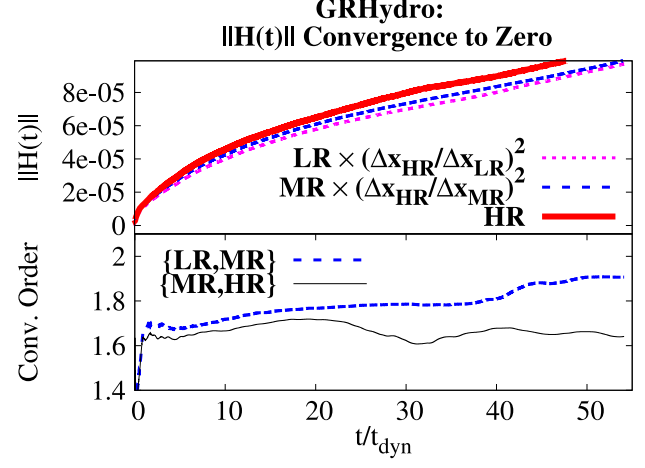

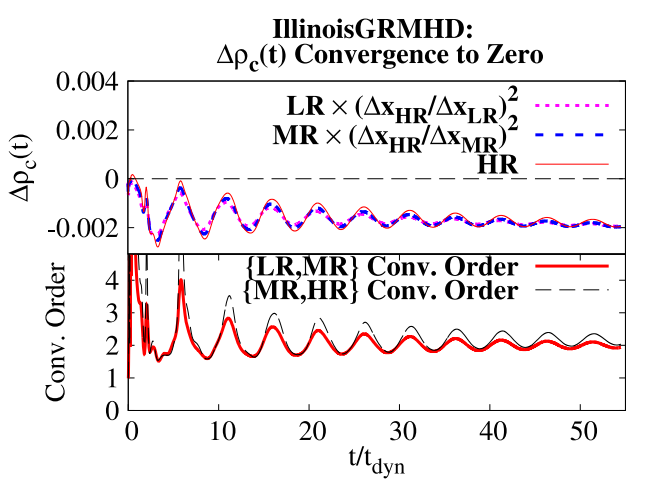

IllinoisGRMHD:

$\|\mathrm{H}(\mathrm{t})\|$ Convergence to Zero

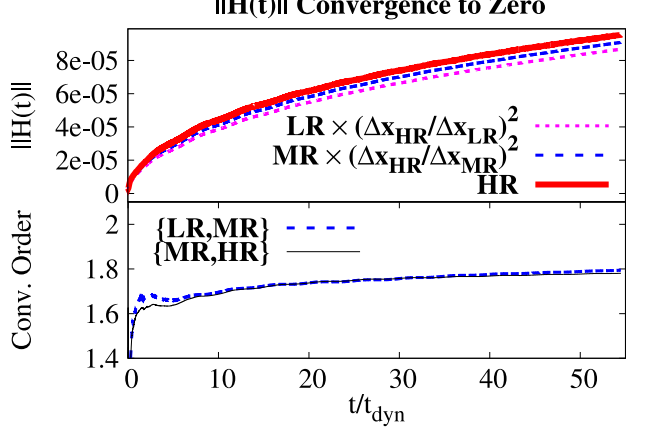

Figure 3. IllinoisGRMHD and GRHydro convergence tests, for dynamic-spacetime, unmagnetized equilibrium TOV star evolutions (with physical and numerical setup as described in appendix C). Upper panels: convergence to zero of TOV star central density drift $\Delta \rho_{c}(t)=\rho_{c}(t) / \rho_{c}(0)-1$, comparing GRHydro (left plot) with IllinoisGRMHD (right plot). Top plots show $\Delta \rho_{c}(t)$ at three separate resolutions, with the low (dotted magenta) and medium (dashed blue) resolution (LR and MR, respectively) simulation results rescaled to high resolution (HR, solid red), assuming that $\Delta \rho_{c}(t)$ converges to zero at second order. Lower plots show implied convergence order to zero (see equation (43)) of $\Delta \rho_{c}(t)$ for pairs of runs, for HR and MR (thin dashed black), and MR and LR (thick solid red), where convergence order to zero is defined as in equation (43). Lower panels: convergence to zero equation (43) of L2 Norm of Hamiltonian constraint violation, $\|H(t)\|$. Top plots show $\|H(t)\|$ at three resolutions, rescaled so that LR (dotted magenta) and MR (dashed blue) results should overlap HR (solid red) results if secondorder convergence to zero is achieved. The bottom plots show implied observed convergence order to zero of pairs of runs: $\mathrm{HR}$ and MR (dashed black), and MR and LR (dashed blue).

GRHydro possesses roughly $8 \%$ higher Hamiltonian constraint violation than IllinoisGRMHD, as shown in figure 4. We conclude that IllinoisGRMHD appears to suffer from less Hamiltonian constraint violation than GRHydro at a given resolution, and exhibits more consistent L2-Norm constraint violation convergence to zero as resolution is increased.

However, at all resolutions, the absolute value of central density drift through 55 dynamical timescales is far higher with IllinoisGRMHD than GRHydro. We analyze the drift at high resolution in the lower panel of figure 4, finding that the differences appear directly after initial settling of the TOV star. These differences are not surprising, given the unique algorithmic choices in each code (e.g., GRHydro adopts the internal energy $\epsilon$ as a primitive 


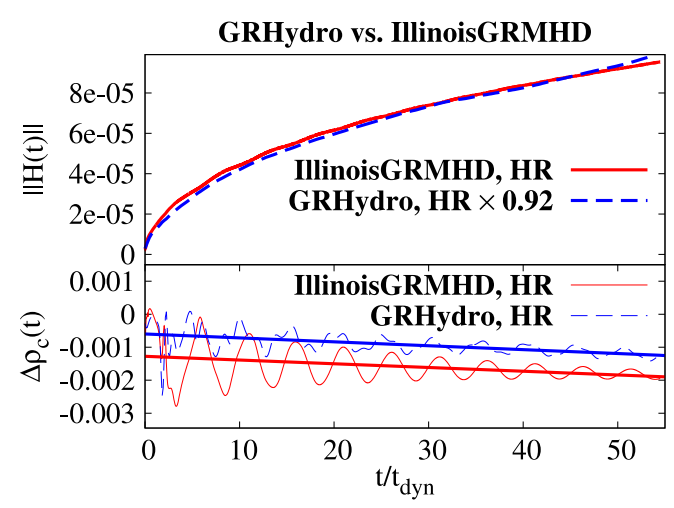

Figure 4. Truncation-error analysis, comparing results from IllinoisGRMHD and GRHydro at high resolution (HR). The top plot shows L2-Norm Hamiltonian constraint violation, for IllinoisGRMHD (red solid) and GRHydro (blue dashed). GRHydro exhibits about $8 \%$ higher constraint violation, so its data were multiplied by 0.92 to achieve a good overlap with IllinoisGRMHD data. The bottom plot shows central density drift $\Delta \rho_{c}(t)=\rho_{c}(t) / \rho_{c}(0)-1$, at high resolution (HR) as well, for IllinoisGRMHD (thin dashed blue) and GRHydro (thin solid red). The thick blue and red lines are linear least-squares fits to IllinoisGRMHD and GRHydro data, from $5 t_{\mathrm{dyn}}$ to $55 t_{\mathrm{dyn}}$. The slope on the GRHydro line is $(-1.19 \pm 0.02) \times 10^{-5}$, and $(-1.13 \pm 0.04) \times 10^{-5}$ for IllinoisGRMHD, where the errors given are standard deviations.

variable, where IllinoisGRMHD adopts pressure $P$ instead, just to name one). In this plot, we fit data in the range $5 \leqslant t / t_{\text {dyn }} \leqslant 55$ to a least-squares linear trendline, finding that the rate of central density drift (i.e., the slope of the linear trendline) after 5 dynamical timescales to be within about one standard deviation for the two codes. In addition, we verified that although the simulation is run for about 2.3 light-crossing times, doubling the outer boundary has no qualitative effect on the results.

In a forthcoming paper, we will demonstrate that differences between these two opensource GRMHD codes spawn from how the GRMHD evolution algorithms are implemented, independent of the chosen reconstruction scheme.

\section{Computational performance benchmarks}

We have demonstrated that although IllinoisGRMHD represents a complete rewrite of OrigGRMHD, the two codes agree to roundoff precision. IllinoisGRMHD is also designed to be more user-friendly, more extensible, and better documented than OrigGRMHD as well. Furthermore, as we demonstrate in this section, IllinoisGRMHD performs and scales better than OrigGRMHD. This stems from the fact that coding decisions within IllinoisGRMHD were made specifically from the outset to optimize not only user-friendliness and code readability, but also performance.

Making IllinoisGRMHD perform as well as GRHydro, on the other hand, appears to be an unlikely goal, as the AMR-capable GRMHD algorithm adopted by IllinoisGRMHD/ OrigGRMHD is far more computationally intensive. All variables in GRHydro's GRMHD scheme for AMR grids (hyperbolic divergence cleaning [19, 50]) are unstaggered, overlapping gridpoints. Meanwhile, IllinoisGRMHD/OrigGRMHD implement a staggered vectorpotential formulation, requiring, e.g., about $60 \%$ more expensive reconstructions to compute 


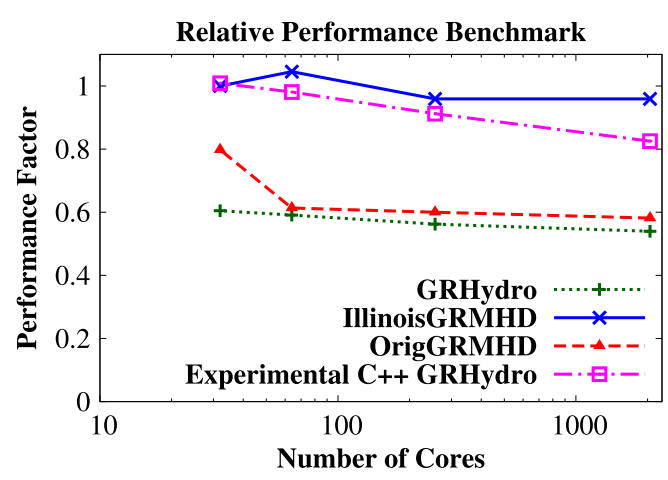

Figure 5. Relative performance of IllinoisGRMHD (blue solid), OrigGRMHD (red dashed), standard GRHydro (green dotted), and the experimental C++ version of GRHydro (magenta dotted-dashed) at multiple problem scales on the Stampede supercluster, performing an unmagnetized neutron star simulation, but with magnetic field evolution enabled. Performance data are normalized to IllinoisGRMHD two-node (32-core) performance (as measured by the number of gridzones computed per second per processor core). As the number of cores was increased, the number of gridpoints per core was kept fixed at approximately $72^{3}$ for all four refined levels and $68^{3}$ for the lowest-resolution level on these AMR grids, effectively making this a weak-scaling test. To convert performance factor to gridzones per second per MPI process (CPU core), simply multiply by 350000 (87 500). Two notes of caution in conversion: First, gridzones per second evaluated is a measure that is highly sensitive to the hardware used and software libraries linked against. Second, Performance Factor is based on the entire code runtime, and a significant fraction of this time measured is spent outside of the GRMHD code, e.g., evolving the spacetime metric and synchronizing ghostzones across MPI processes, just to name a couple.

GRMHD fluxes, as they must be computed on staggered gridpoints. In addition, the evolution of the staggered EM vector potential gauge quantity $[\sqrt{\gamma} \Phi]$ is quite expensive, as it requires a large number of interpolations. Of course, in exchange for this more expensive algorithm is the guarantee that monopoles (i.e., violations of $\nabla \cdot \boldsymbol{B}=0$ ) cannot be generated on grid refinement boundaries when magnetized fluid flows cross them. GRHydro cannot guarantee this, but IllinoisGRMHD/OrigGRMHD does.

Thus a priori, we would expect IllinoisGRMHD and OrigGRMHD to significantly under-perform GRHydro. Remarkably, figure 5 demonstrates that for a physical system and AMR grid hierarchy typically used in production runs, IllinoisGRMHD actually outperforms both GRHydro and OrigGRMHD by a comfortable margin. There also exists a new, experimental C++ version of GRHydro (henceforth, GRHydro-experimental), which was written in part to improve performance. Indeed, performance is greatly enhanced by GRHydroexperimental, but it at best matches IllinoisGRMHD performance at small core counts, and scales worse than IllinoisGRMHD with increasing core count. The physical system and basic AMR grid hierarchy is as described in appendix C; i.e., it consists of an unmagnetized, equilibrium TOV star for which the magnetic-field is also evolved but initialized to zero.

As measured by the number of gridpoints computed per second per core on the TACC Stampede supercomputer, at all problem scales typically used for parallel AMR runs (ranging from 32 to 2048 cores), figure 5 shows that IllinoisGRMHD consistently outperforms the standard GRHydro by a factor of between 1.7 and 1.8. However, IllinoisGRMHD matches GRHydro-experimental's performance, to within measurement error at 32 cores, but manages to outperform GRHydro-experimental by about $16 \%$ at 2048 cores. Again, it is remarkable 


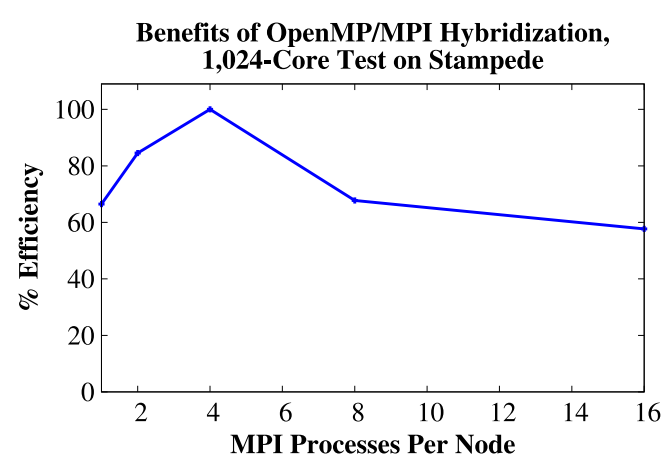

Figure 6. IllinoisGRMHD code performance as the number of MPI processes per node on Stampede is varied, with the total core-count fixed at 1024 (i.e., 64 Stampede nodes). When running with $1,2,4,8$, and 16 MPI processes per node, the number of threads per MPI process (OMP_NUM_THREADS) was set to $16,8,4,2$, and 1 , respectively. Efficiency is normalized to the 4 MPI processes per node case. Simulation is of a neutron star with full GRMHD and spacetime evolution enabled.

that IllinoisGRMHD can produce performance numbers in the same ballpark as GRHydro, as IllinoisGRMHD implements a much more expensive GRMHD algorithm.

The performance improvement over OrigGRMHD is also significant, with IllinoisGRMHD outperforming OrigGRMHD by a factor of 1.3 at 32 cores, increasing to 1.6 at 256 and 2048 cores. In independent testing, we find that about 10-20\% of the performance difference between IllinoisGRMHD and OrigGRMHD is due to the fact that OrigGRMHD is based on an old, unmaintained version of the Cactus/Carpet infrastructure (ca. October, 2010). For more details on the physical system and basic grid structure of this benchmark, see appendix C.

Benchmarks presented here measure total simulation performance, and since these are dynamical spacetime simulations, the performance gap between IllinoisGRMHD and the other codes will certainly increase for fixed-background-spacetime simulations. Thus by adopting IllinoisGRMHD, research groups currently using the standard version of GRHydro or OrigGRMHD stand to boost their computational resources by a factor of between 1.6 and 1.8. Independent tests indicate that the performance gap increases to a factor of $\approx 2$ in fixedspacetime-background GRMHD simulations.

Making AMR-based codes like IllinoisGRMHD, OrigGRMHD, and GRHydro scale well is an intrinsically difficult task. AMR greatly reduces the memory and processor overhead in our simulations, focusing resolution only where it is needed, and generating many small, refined numerical grids in the process. When these small refined grids are parallelized, however, they are generally split into even smaller grids, resulting in a large grid surface area to volume ratio. As the information on the surfaces must be communicated across nodes, this makes the performance of AMR-based codes strongly network-limited.

OpenMP ${ }^{10}$ can be used to combat this by splitting computational loops over multiple processor cores, enabling us to use fewer parallel (MPI) processes per CPU and thus larger grids on a given (MPI) process. This reduces the network load significantly and thus increases overall performance .

As shown in figure 6, in production-scale benchmarks, we find that IllinoisGRMHD performs slightly more than $40 \%$ faster as an OpenMP/MPI hybrid code than as a pure MPI code (i.e., when running 16 MPI processes per node, OpenMP disabled), for a typical

10 OpenMP Architecture Review Board. OpenMP application program interface version 3.0, 2008. 
GRMHD production run on the Stampede supercluster. Although all three codes possess some degree of OpenMP support, all of IllinoisGRMHD's loops have has been written with full OpenMP (see footnote 8) support, making IllinoisGRMHD a pure OpenMP/MPI hybrid code, just like OrigGRMHD. We finish this section by noting that all benchmark results in figure 5 were performed using 4 MPI processes per node and 4 OpenMP threads per MPI process, which we found maximized performance in all codes.

\section{Conclusions and future work}

The field of numerical relativity has matured considerably in the years since the first dynamical spacetime GRMHD codes were developed, and multiple groups now possess such codes. Given that the future of our field depends on the ability to advance and extend these codes to model new physics, while still maintaining and improving the GRMHD modules, it stands to reason that the community could benefit if we consolidated our efforts and adopted the same dynamic-spacetime GRMHD code.

With its proven robustness and reliability in modeling some of the most extreme phenomena in the Universe, it seems the OrigGRMHD code could be a good candidate for such community adoption if it were open-sourced. But despite its strong scientific track record, OrigGRMHD was not written with community adoption in mind, instead being a code written 'by experts and for experts' of OrigGRMHD, with a premium put on immediate applications. As such, the code lacked a number of features common to large, open-source, communitybased codes in computational astrophysics, including sufficient documentation and code comments, fine-grained modularity, a consistent coding style, and regular, enforced code maintenance (e.g., removal of unused and unmaintained features).

As an open-source, from-the-ground-up rewrite of OrigGRMHD, IllinoisGRMHD aims to fix all the former code's idiosyncracies, thus facilitating widespread community adoption. With such adoption in mind, IllinoisGRMHD's development has been guided by the four core design principles of user-friendliness, modularity/extensibility, robustness, and performance/ scalability. Regarding user-friendliness, the code is well-documented, properly commented, and requires only basic programming skills to understand and run. IllinoisGRMHD is also far more modular and extensible than OrigGRMHD, with low-level computational fluid dynamics routines split off from the main code into a library of extensible functions.

As for robustness, IllinoisGRMHD was designed to act as a drop-in replacement for OrigGRMHD, and we have demonstrated that IllinoisGRMHD indeed reproduces results from the original code to roundoff-level precision, not only when evolving magnetized NSs, but also discontinuous, random initial data.

In addition, IllinoisGRMHD largely produces consistent results with the only other opensource, dynamical spacetime GRMHD code, GRHydro, in that both codes exhibit approximate second-order convergence. Although both codes were run with the same basic evolution algorithms, results differ due to the specific details of how these algorithms were implemented. We will explore this further in a forthcoming work, but just to name a couple of differences, GRHydro reconstructs the specific internal energy $\epsilon$ and the Valencia-formulation three-velocity, while IllinoisGRMHD reconstructs pressure and three-velocity defined as $v^{i}=u^{i} / u^{0}$. When evolving equilibrium unmagnetized TOV stars, GRHydro produces about $8 \%$ higher Hamiltonian constraint violations (as measured by the L2 Norm over the entire grid), but significantly less absolute central density drift than IllinoisGRMHD. We find that the rate of central density drift is identical between the two codes after the star undergoes an initial settling over a few dynamical timescales. We conclude it is this initial settling that causes the large discrepancy in absolute central density drift. 
Though user-friendliness, modularity/extensibility, and robustness were the primary considerations in IllinoisGRMHD's development, it would be hard to convince key developers of other codes to adopt IllinoisGRMHD unless we could demonstrate at least comparable performance and scalability to alternative dynamical spacetime GRMHD codes. To this end, we have shown that IllinoisGRMHD is in fact about 1.7-1.8 times faster than the standard version of GRHydro for production-size, AMR-enabled, GRMHD runs on the Stampede supercluster, scaling to typical high-resolution core-counts at better than $95 \%$ efficiency.

This is a rather remarkable result, as IllinoisGRMHD implements a far more computationally expensive GRMHD algorithm than GRHydro to ensure the no-monopole constraint $\nabla \cdot \boldsymbol{B}=0$ is satisfied. This added expense forbids the generation of monopoles at AMR grid boundaries in the case of multi-scale GRMHD flows, which GRHydro, and even its new, experimental $\mathrm{C}++$ version, which we refer to as GRHydro-experimental, cannot guarantee. GRHydro-experimental, which was actively being written during the preparation of this paper, is a complete rewrite of the standard, FORTRAN-based GRHydro, with a goal in part of improving performance. Indeed, GRHydro-experimental does improve performance, but only at best matches IllinoisGRMHD's performance at small core counts, with IllinoisGRMHD performing about $16 \%$ better at 1024-2048 cores. Though these results are based on a single scenario and may change slightly when simulating other systems of interest, we consider them illustrative of what one should expect.

IllinoisGRMHD also outperforms OrigGRMHD by a factor of 1.3-1.6. Thus by adopting IllinoisGRMHD, research groups using GRHydro or OrigGRMHD stand to increase their computational resources available for dynamical spacetime, GRMHD runs. While in terms of performance, IllinoisGRMHD seems to be only slightly better than the experimental, C++ version of GRHydro, perhaps the two greatest advantages of IllinoisGRMHD over GRHydro is that (1) IllinoisGRMHD does not allow the generation of magnetic monopoles when modeling multi-scale GRMHD flows on AMR grids and (2) IllinoisGRMHD is capable of stably modeling GRMHD flows into BH horizons over very long timescales, without the need for special algorithms that excise GRMHD data with the BH. GRHydro requires excision to model such flows and its GRMHD features have been mostly used for core collapse (to a NS) simulations, in which no BH is present. We conclude that making GRHydro's GRMHD schemes as robust may require careful specification of boundary conditions on the excision surface coupled to an interpolation scheme across AMR level boundaries that respects the nomonopoles constraint.

As mentioned previously, a forthcoming paper will analyze how differences in algorithmic implementations between GRHydro and IllinoisGRMHD can lead to significantly different results when evolving NSs. One possibility is that we may find an implementation that results in a superior code to either original code. If such a code is found, it may prove quite useful to reliably evolving binary NSs and BH-NSs over many orbits with a minimum of computational expense.

Although IllinoisGRMHD is ready for production runs now, we encourage other developers to join our effort in improving IllinoisGRMHD beyond its current state, as a great deal of important work remains to be done. We would like to port features from GRHydro into IllinoisGRMHD's library of functions, using IllinoisGRMHD's coding style, including reconstruction schemes, conservative-to-primitives solvers, and more advanced approximate Riemann solvers, just to name a few. IllinoisGRMHD was originally written in a standalone sandbox for maximum portability, and was only recently ported into the ET. It therefore makes minimal use of certain aspects of the ET infrastructure that could greatly extend its usefulness. For example, the current version supports only single gamma-law EOSs, and full 
3D simulations with either no symmetries enabled, or simply bitant symmetry across the $x y$-plane. The ET infrastructure provides support for arbitrary EOSs, symmetry conditions, etc, and we intend to work with the large ET community toward making this code the standard choice in the ET, and one the community can be proud of.

\section{Acknowledgments}

We gratefully acknowledge the ET community for hosting the IllinoisGRMHD software, and Sean T McWilliams for useful discussions and for generously lending time on the Spruce Knob HPC resource at WVU. This paper was supported in part by NSF Grant PHY-1300903 and NASA Grants NNX13AH44G and 13-ATP13-0077. VP is supported in part by the Simons Foundation and by NSF grant PHY-1305682. This work used the Extreme Science and Engineering Discovery Environment (XSEDE), which is supported by NSF grant number OCI-1053575.

\section{Appendix A. Physical and computational setup for magnetized TOV star code validation tests}

The magnetized TOV star used in these tests is precisely the same stable neutron star as described in appendix $\mathrm{C}$, except it is seeded with a weak magnetic field at $t=0$. This initial magnetic field is purely poloidal, with vector potential components defined as

$$
\begin{aligned}
& A_{x}=-y A_{b} \max \left(P-P_{\text {cut }}, 0\right), \\
& A_{y}=x A_{b} \max \left(P-P_{\text {cut }}, 0\right), \\
& A_{z},[\sqrt{\gamma} \Phi]=0,
\end{aligned}
$$

where $P_{\text {cut }}$ is set to $4 \%$ of the maximum pressure and $A_{b}$ is set so that the maximum initial magnetic-to-gas pressure ratio $\beta^{-1}$ is $0.83 \times 10^{-3}$. Like the convergence tests, these tests are dynamical spacetime tests, but unlike the convergence tests, IllinoisGRMHD and OrigGRMHD codes are each coupled to the (closed-source) BSSN dynamical spacetime module of the Illinois NR group. Further, the initial TOV star density, pressure, and spacetime metric profiles are provided by the code of [18], which generates data for this nonrotating star that agrees to machine precision with the TOVSolver code used in the unmagnetized tests.

These tests are also performed on AMR grids, with the coarsest grid cube extended so its half-side-length is $10 R_{\mathrm{NS}}$, centered on the NS. Two finer AMR grid levels are nested within this coarse grid: the next finer grid having half-side-length $5 R_{\mathrm{NS}}$ and the finest having $2.5 R_{\mathrm{NS}}$. Again, at each finer level, the grid spacing is halved, so that the cube with half-side-length $2.5 R_{\mathrm{NS}}$ has a grid spacing of $\Delta x \approx\{0.078\} R_{\mathrm{NS}}$. This corresponds to resolving the NS to 26 gridpoints across its diameter. The resolution is intentionally set to be very low, to guarantee that truncation-error differences between the codes will be more strongly magnified than at (higher) resolutions typically chosen for evolving NSs. In addition, a close outer boundary is chosen to maximize its influence on the evolution, to check for errors in coding the outflow outer boundary conditions. Finally, we choose our low-density atmosphere density floor to correspond to $10^{-7}$ times the initial central density of the NS. 
Table B1. Prescription for setting GRMHD and spacetime metric quantities in random initial data module. Note that the initial data employ the polytropic EOS $P=\rho_{0}^{2}$, and we evolve with the gamma-law EOS $P=(\Gamma-1) \rho_{0} \epsilon$ with $\Gamma=2$.

\begin{tabular}{lc}
\hline Variable & Value set in random initial data module \\
\hline$\rho_{0}$ & $0.01 S$ \\
$P$ & $\rho_{0}^{2}$ (Gamma-law EOS) \\
$\phi$ & $0.1 S$ \\
$\alpha$ & $1-0.1 S$ \\
$\left\{v^{x}, v^{y}, v^{z}\right\}$ & $\{1,1.1,0.9\} \times 10^{-1} \xi$ \\
$\left\{[\sqrt{\gamma} \Phi], A_{x}, A_{y}, A_{z}\right\}$ & $\{0.6,1,1.1,0.9\} \times 10^{-1} \xi$ \\
$\left\{\beta^{x}, \beta^{y}, \beta^{z}\right\}$ & $\{0.9,1.1,1\} \times 10^{-1} \xi$ \\
$\left\{\tilde{\gamma}_{x x}, \tilde{\gamma}_{y y}, \tilde{\gamma}_{z z}\right\}$ & $1+\{10,1,10\} \times 10^{-3} \xi$ \\
$\left\{\tilde{\gamma}_{x y}, \tilde{\gamma}_{x z}, \tilde{\gamma}_{y z}\right\}$ & $\{1,10,1\} \times 10^{-4} \xi$ \\
$\left\{K_{x x}, K_{x y}, K_{x z}, K_{y y}, K_{y z}, K_{z z}\right\}$ & $\{10,2,3,40,5,60\} \times 10^{-3} \xi$ \\
\hline
\end{tabular}

\section{Appendix B. Random initial data}

The algorithm used within our random initial data module is as follows. At a given gridpoint, the random number generator is first seeded with a unique integer based on the coordinate of the gridpoint. The seed is then used to generate one double-precision random number $\xi \in(-0.1,0.1)$. Because this random number is based entirely on the coordinate of a gridpoint, the initial data will be consistent when evolving on identical grids, regardless of how the global grid is split among processors when generating and evolving initial data in a parallel run.

Using this double-precision random number $\xi \in(-0.1,0.1)$, we define

$$
S=\xi+\frac{1}{7}[4+\sin (10 x / L)+\sin (10 y / L)+\cos (10 z / L)]
$$

where $L$ denotes the coarsest AMR grid cube half-side-length. Thus $S \in\left(\frac{3}{70}, 1.1\right)$ consists of a strong stochastic perturbation atop a smoothly oscillating function. Given the quantities $S$ and $\xi$, all basic spacetime metric and GRMHD quantities may then be set following the prescription detailed in table B1.

Although the resulting data are not designed to satisfy Einstein's equations, be conformally flat, or be consistent with the BSSN formalism, we make sure the spacetime metric is Lorentzian and the three-metric positive definite. We also enforce the BSSN constraint $\tilde{\gamma}=1$ as follows. Immediately after the quantities in table B1 have been set at a given gridpoint, we compute the resulting non-unit determinant $\tilde{\gamma}$ and then multiply the components of $\tilde{\gamma}_{i j}$ by $\tilde{\gamma}^{-1 / 3}$. In this way, the unit determinant of the three-metric is enforced. After applying this constraint, all GRMHD and spacetime metric quantities not specified in table B1 (e.g., GRMHD conservative variables, $B^{i}$, $\tilde{\gamma}^{i j}$, etc) are directly computed from quantities in that table.

In tests adopting this random initial data module, we choose an AMR grid with one refinement level centered at the origin. The coarser grid, with grid spacing 1.0, has cube halfside-length of 10, and the finer grid, with grid spacing 0.5, has grid half-side-length of 2 . Space-time evolution modules, as well as prolongation and restriction operations on spacetime variables, are disabled. 
The initial maximum rest-mass density is chosen to be $\sim 10 \%$ the rest-mass density of the TOV star used in other tests. The atmospheric density floor is set to $10^{-6}$, which is about $10^{-4}$ times the maximum possible initial density, yielding stochastic fluctuations in density spanning about four orders of magnitude. Additionally, the magnetic-to-gas-pressure ratio at $t=0$ ranges from $\sim 10^{-3}$ (gas-pressure dominated) to about 10 (magnetic-pressure dominated) initially. Given that initial data at a given gridpoint are independently and randomly specified, we conclude that physical quantities at neighboring gridpoints can differ by several orders of magnitude, making this a very harsh test. Despite the lack of coherent GRMHD flows, throughout the evolution of these random initial data, the Lorentz factor limit of $W=10$ is exceeded and subsequently enforced about 400 times.

\section{Appendix C. Physical and computational setup for unmagnetized TOV star convergence tests}

IllinoisGRMHD and GRHydro evolve unmagnetized, polytropic TOV star initial data, consisting of a TOV star with central density of 0.129285309 constructed with a polytropic equation of state $P=K \rho^{\Gamma}$, where $K=1$ and $\Gamma=2$. This generates a model for a cold, degenerate NS with compaction $M_{\mathrm{NS}} / R_{\mathrm{NS}} \approx 0.1467$. The initial data are generated using the built-in TOVSolver thorn within the ET. The convergence tests are dynamical spacetime tests, in which IllinoisGRMHD and GRHydro are each coupled to the McLachlan BSSN thorn. To match IllinoisGRMHD's evolution algorithms, the 'HLLE' approximate Riemann solver is chosen for GRHydro evolutions, along with PPM reconstruction. Though the codes differ in their usage of these algorithms (e.g., GRHydro reconstructs the internal energy $\epsilon$ instead of pressure $P$, etc), the parameters are chosen so that both codes share precisely the same algorithms. Note that the outer boundary conditions for the spacetime variables are set to be identical between the two codes, but the hydrodynamic boundary conditions differ. However, this is inconsequential, as the density near the outer boundary remains within $\approx 1 \%$ of the original atmosphere value in both codes throughout the entire evolution.

These tests are performed on cubic AMR grids, so that the coarsest grid cube possesses a half-side-length of $40 R_{\mathrm{NS}}$, centered on the NS. The AMR hierarchy-nested within this coarse grid and also centered on the NS-consists of four, progressively higher-resolution cubes with half-side-lengths of $15 R_{\mathrm{NS}}, 7.5 R_{\mathrm{NS}}, 3.75 R_{\mathrm{NS}}$, and $1.875 R_{\mathrm{NS}}$. At each finer level, the grid spacing is halved, so that the cube with half-side-length $1.875 R_{\mathrm{NS}}$ has a grid spacing of $\Delta x=\{0.03906,0.03125,0.025\} R_{\mathrm{NS}}$ for low, medium, and high-resolution runs, respectively. This corresponds to resolving the NS across its diameter to approximately 51, 64, and 80 gridpoints, for low, medium, and high-resolution runs, respectively. In both codes, lowdensity atmosphere density floor is set to correspond to $10^{-7}$ times the initial central density of the NS.

\section{References}

[1] Alic D, Bona-Casas C, Bona C, Rezzolla L and Palenzuela C 2012 Conformal and covariant formulation of the Z4 system with constraint-violation damping Phys. Rev. D 85064040

[2] Anderson M, Hirschmann E, Liebling S L and Neilsen D 2006 Relativistic MHD with adaptive mesh refinement Class. Quantum Grav. 23 6503-24

[3] Anninos P and Fragile P C 2003 Nonoscillatory central difference and artificial viscosity schemes for relativistic hydrodynamics Astrophys. J. Suppl. 144 243-57 
[4] Antón L, Zanotti O, Miralles J A, Martí J M, Ibáñez J M, Font J A and Pons J A 2006 Numerical 3 +1 general relativistic magnetohydrodynamics: a local characteristic approach Astrophys. J. 637 $296-312$

[5] Arnowitt R, Deser S and Misner C W 1962 Gravitation: An Introduction to Current Research (New York: Wiley) pp 227-65

[6] Baiotti L, Hawke I, Montero P and Rezzolla L 2003 A new three-dimensional general-relativistic hydrodynamics code Computational Astrophysics in Italy: Methods and Tools ed R Capuzzo-Dolcetta vol 1 (Trieste: MSAIt) p 210

[7] Baiotti L, Hawke I, Montero P J, Löffler F, Rezzolla L, Stergioulas N, Font J A and Seidel E 2005 Three-dimensional relativistic simulations of rotating neutron star collapse to a Kerr black hole Phys. Rev. D 71024035

[8] Baiotti L, Hawke I, Montero P J and Rezzolla L 2003 A new three-dimensional general-relativistic hydrodynamics code Mem. Soc. Astron. Ital. 1 210-9

[9] Balsara D S 2001 Divergence-free adaptive mesh refinement for magnetohydrodynamics J. Comput. Phys. 174 614-48

[10] Balsara D S 2009 Divergence-free reconstruction of magnetic fields and WENO schemes for magnetohydrodynamics J. Comput. Phys. 228 5040-56

[11] Balsara D S and Spicer D S 1999 A staggered mesh algorithm using high order godunov fluxes to ensure solenoidal magnetic fields in magnetohydrodynamic simulations J. Comput. Phys. 149 270-92

[12] Banyuls F, Font J A, Ibáñez J M M, Martí J M and Miralles J A 1997 Numerical \{3 + 1$\}$ general relativistic hydrodynamics: a local characteristic approach Astrophys. J. 476221

[13] Baumgarte T W and Shapiro S L 1998 Numerical integration of Einstein's field equations Phys. Rev. D 59024007

[14] Baumgarte T W and Shapiro S L 2010 Numerical Relativity: Solving Einstein's Equations on the Computer (Cambridge: Cambridge University Press)

[15] Brown J D, Diener P, Sarbach O, Schnetter E and Tiglio M 2009 Turduckening black holes: an analytical and computational study Phys. Rev. D 79044023

[16] Cerdá-Durán P, Font J A, Antón L and Müller E 2008 A new general relativistic magnetohydrodynamics code for dynamical spacetimes Astron. Astrophys. 492 937-53

[17] Colella P and Woodward P R 1984 The piecewise parabolic method (PPM) for gas-dynamical simulations J. Comput. Phys. 54 174-201

[18] Cook G B, Shapiro S L and Teukolsky S A 1994 Rapidly rotating polytropes in general relativity Astrophys. J. 422 227-42

[19] Dedner A, Kemm F, Kröner D, Munz C-D, Schnitzer T and Wesenberg M 2002 Hyperbolic divergence cleaning for the MHD equations J. Comput. Phys. 175 645-73

[20] Del Zanna L, Bucciantini N and Londrillo P 2003 An efficient shock-capturing central-type scheme for multidimensional relativistic flows: II. Magnetohydrodynamics Astron. Astrophys. 400 397-413

[21] Dionysopoulou K, Alic D, Palenzuela C, Rezzolla L and Giacomazzo B 2013 General-relativistic resistive magnetohydrodynamics in three-dimensions: formulation and tests Phys. Rev. D $\mathbf{8 8}$ 044020

[22] Duez M D, Liu Y T, Shapiro S L, Shibata M and Stephens B C 2006 Collapse of magnetized hypermassive neutron stars in general relativity Phys. Rev. Lett. 96031101

[23] Duez M D, Liu Y T, Shapiro S L, Shibata M and Stephens B C 2006 Evolution of magnetized, differentially rotating neutron stars: simulations in full general relativity Phys. Rev. D 73 104015

[24] Duez M D, Liu Y T, Shapiro S L and Stephens B C 2005 Excitation of magnetohydrodynamic modes with gravitational waves: a testbed for numerical codes Phys. Rev. D 72024029

[25] Duez M D, Liu Y T, Shapiro S L and Stephens B C 2005 Relativistic magnetohydrodynamics in dynamical spacetimes: numerical methods and tests Phys. Rev. D 72024028

[26] East W E, Pretorius F and Stephens B C 2012 Hydrodynamics in full general relativity with conservative AMR Phys. Rev. D 85124010

[27] Etienne Z B, Faber J A, Liu Y T, Shapiro S L, Taniguchi K and Baumgarte T W 2008 Fully general relativistic simulations of black hole-neutron star mergers Phys. Rev. D 77084002

[28] Etienne Z B, Liu Y T, Paschalidis V and Shapiro S L 2012 General relativistic simulations of black-hole-neutron-star mergers: effects of magnetic fields Phys. Rev. D 85064029 
[29] Etienne Z B, Liu Y T and Shapiro S L 2006 General relativistic simulations of slowly and differentially rotating magnetized neutron stars Phys. Rev. D 74044030

[30] Etienne Z B, Liu Y T and Shapiro S L 2010 Relativistic magnetohydrodynamics in dynamical spacetimes: a new adaptive mesh refinement implementation Phys. Rev. D 82084031

[31] Etienne Z B, Liu Y T, Shapiro S L and Baumgarte T W 2009 General relativistic simulations of black-hole-neutron-star mergers: effects of black-hole spin Phys. Rev. D 79044024

[32] Etienne Z B, Paschalidis V, Liu Y T and Shapiro S L 2012 Relativistic magnetohydrodynamics in dynamical spacetimes: improved electromagnetic gauge condition for adaptive mesh refinement grids Phys. Rev. D 85024013

[33] Etienne Z B, Paschalidis V and Shapiro S L 2012 General-relativistic simulations of black-holeneutron-star mergers: effects of tilted magnetic fields Phys. Rev. D 86084026

[34] Evans C R and Hawley J F 1988 Simulation of magnetohydrodynamic flows-a constrained transport method Astrophys. J. 332 659-77

[35] Fan X, Messenger C and Heng I S 2014 A Bayesian approach to multi-messenger astronomy: identification of gravitational-wave host Galaxies Astrophys. J. 79543

[36] Farris B D, Gold R, Paschalidis V, Etienne Z B and Shapiro S L 2012 Binary black-hole mergers in magnetized disks: simulations in full general relativity Phys. Rev. Lett. 109221102

[37] Farris B D, Liu Y T and Shapiro S L 2011 Binary black hole mergers in gaseous disks: Simulations in general relativity Phys. Rev. D 84024024

[38] Gammie C F, McKinney J C and Tóth G 2003 HARM: a numerical scheme for general relativistic magnetohydrodynamics Astrophys. J. 589 444-57

[39] Giacomazzo B and Rezzolla L 2007 WhiskyMHD: a new numerical code for general relativistic magnetohydrodynamics Class. Quantum Grav. 24 S235

[40] Gold R, Paschalidis V, Etienne Z B, Shapiro S L and Pfeiffer H P 2014 Accretion disks around binary black holes of unequal mass: general relativistic magnetohydrodynamic simulations near decoupling Phys. Rev. D 89064060

[41] Gold R, Paschalidis V, Ruiz M, Shapiro S L, Etienne Z B and Pfeiffer H P 2014 Accretion disks around binary black holes of unequal mass: GRMHD simulations of postdecoupling and merger Phys. Rev. D 90104030

[42] Harten A, Lax P D and van Leer B 1983 On upstream differencing and godunov-type schemes for hyperbolic conservation laws SIAM Rev. 2535

[43] Janka H-T, Zwerger T and Moenchmeyer R 1993 Does artificial viscosity destroy prompt type-II supernova explosions? Astron. Astrophys. 268 360-8

[44] Kiuchi K, Kyutoku K and Shibata M 2012 Three-dimensional evolution of differentially rotating magnetized neutron stars Phys. Rev. D 86064008

[45] LIGO Scientific Collaboration et al 2012 Implementation and testing of the first prompt search for gravitational wave transients with electromagnetic counterparts Astron. Astrophys. 539 A 124

[46] Liu Y T, Shapiro S L, Etienne Z B and Taniguchi K 2008 General relativistic simulations of magnetized binary neutron star mergers Phys. Rev. D 78024012

[47] Liu Y T, Shapiro S L and Stephens B C 2007 Magnetorotational collapse of very massive stars to black holes in full general relativity Phys. Rev. D 76084017

[48] Löffler F et al 2012 The Einstein Toolkit: a community computational infrastructure for relativistic astrophysics Class. Quantum Grav. 29115001

[49] Mart J M, Ibáez J M and Miralles J A 1991 Numerical relativistic hydrodynamics: local characteristic approach Phys. Rev. D 43 3794-801

[50] Mösta P, Mundim B C, Faber J A, Haas R, Noble S C, Bode T, Löffler F, Ott C D, Reisswig C and Schnetter E 2014 GRHydro: a new open-source general-relativistic magnetohydrodynamics code for the Einstein toolkit Class. Quantum Grav. 31015005

[51] Nissanke S, Sievers J, Dalal N and Holz D 2011 Localizing compact binary inspirals on the sky using ground-based gravitational wave interferometers Astrophys. J. 73999

[52] Noble S C, Gammie C F, McKinney J C and del Zanna L 2006 Primitive variable solvers for conservative general relativistic magnetohydrodynamics Astrophys. J. 641 626-37

[53] Noble S C, Krolik J H and Hawley J F 2009 Direct calculation of the radiative efficiency of an accretion disk around a black hole Astrophys. J. 692 411-21

[54] Palenzuela C 2013 Modelling magnetized neutron stars using resistive magnetohydrodynamics Mon. Not. R. Astron. Soc. 431 1853-65 
[55] Paschalidis V, Etienne Z B, Liu Y T and Shapiro S L 2011 Head-on collisions of binary white dwarf-neutron stars: simulations in full general relativity Phys. Rev. D 83064002

[56] Paschalidis V, Etienne Z B and Shapiro S L 2012 Importance of cooling in triggering the collapse of hypermassive neutron stars Phys. Rev. D 86064032

[57] Paschalidis V, Etienne Z B and Shapiro S L 2013 General-relativistic simulations of binary black hole-neutron stars: precursor electromagnetic signals Phys. Rev. D 88021504

[58] Paschalidis V, Liu Y T, Etienne Z B and Shapiro S L 2011 Merger of binary white dwarf-neutron stars: simulations in full general relativity Phys. Rev. D 84104032

[59] Paschalidis V, Ruiz M and Shapiro S L 2015 Relativistic simulations of black hole-neutron star coalescence: the jet emerges Astrophys. J. Lett. 806 L14

[60] Paschalidis V and Shapiro S L 2013 A new scheme for matching general relativistic ideal magnetohydrodynamics to its force-free limit Phys. Rev. D 88104031

[61] Ruiz M, Paschalidis V and Shapiro S L 2014 Pulsar spin-down luminosity: simulations in general relativity Phys. Rev. D 89084045

[62] Schnetter E, Hawley S H and Hawke I 2004 Evolutions in 3D numerical relativity using fixed mesh refinement Class. Quantum Grav. 21 1465-88

[63] Sekiguchi Y, Kiuchi K, Kyutoku K and Shibata M 2012 Current status of numerical-relativity simulations in Kyoto Prog. Theor. Exp. Phys. 1 A304

[64] Shah S, van der Sluys M and Nelemans G 2012 Using electromagnetic observations to aid gravitational-wave parameter estimation of compact binaries observed with LISA Astron. Astrophys. 544 A153

[65] Shibata M, Duez M D, Liu Y T, Shapiro S L and Stephens B C 2006 Magnetized hypermassive neutron-star collapse: a central engine for short gamma-ray bursts Phys. Rev. Lett. 96031102

[66] Shibata M, Liu Y T, Shapiro S L and Stephens B C 2006 Magnetorotational collapse of massive stellar cores to neutron stars: simulations in full general relativity Phys. Rev. D 74104026

[67] Shibata M and Nakamura T 1995 Evolution of three-dimensional gravitational waves: harmonic slicing case Phys. Rev. D 52 5428-44

[68] Stephens B C, Duez M D, Liu Y T, Shapiro S L and Shibata M 2007 Collapse and black hole formation in magnetized, differentially rotating neutron stars Class. Quantum Grav. 24207 